\title{
The Value of Learning in the Product Development Stage: A Real Options Approach
}

\author{
Jeffrey Sadowsky \\ Stanford University*
}

February 2, 2005

\begin{abstract}
In this paper we use a real options approach to value pilot project investments that help reduce idiosyncratic uncertainty with respect to the final costs of a project. We develop a general one period investment model and, using standard financial engineering techniques, are able to find the value of these investment opportunities and the corresponding optimal investment level. In our setting, both tradable market uncertainty and idiosyncratic technical uncertainty affect the value of the project, with the latter being driven by the amount invested in the pilot stage. Learning is modeled using a proportionality assumption between investment in the development stage and resolution of technical uncertainty. The coefficient that governs this proportionality relation will play a key role in our model, as it defines whether there are decreasing or increasing marginal returns to investment in the development stage and to what extent. Interesting economic implications concerning the effects of this learning coefficient and other parameters of interest in the optimal investment decision are obtained. The robustness of our results is also analyzed. Finally, applications of our framework to investment decisions in various industries and across the supply chain of firms are discussed.
\end{abstract}

*Dept. of Management Science and Engineering, Terman Building Room 477. Email:sadowsky@stanford.edu. This work was done under the supervision of Prof. Blake Johnson from Stanford University. The author also wishes to thank James Primbs, John Weyant, Eymen Errais, as well as Scott Mathews from the Boeing Corporation for helpful discussions 


\section{Introduction}

For a firm, optimal allocation of resources during the development stage of a product is a complex task, more so in the presence of significant market uncertainty, as the lag of these investments with respect to the future cash flows received in case of successful completion is considerable. In technologically intensive industries the problem is even more daunting, as the final product could require more than a few different technological systems working together and the total funding devoted to $R \& D$ must be split between different technologies. Technical learning and the possibility of launching a profitable commercial stage after the development stage is completed, are the main sources of value of these $R \& D$ investment opportunities. We will define technical learning in the most general way as the reduction of uncertainty with respect to the efficient technological frontier of a firm. Generally, this source of risk is idiosyncratic, i.e., specific to the firm or the particular project under consideration. Keeping market prices constant, a positive evolution of this technical uncertainty will increase the final output/input ratio of the firm, thus, increasing the value of the project under consideration and viceversa. However, market factors are not constant. In fact, they are significant drivers of the value of most projects. These market factors evolve through time, making learning more or less valuable in different scenarios. Unlike technical uncertainty, which can be reduced by investing in the development stage of a product, market uncertainty is mostly beyond the control of the firm and, at least somewhat correlated with economic fundamentals.

The purpose of our work is to give a simple Real Options based framework to value investment opportunities that have two main purposes: they make feasible the launch of the commercial stage of a project, giving the firm the possibility of future payoffs, and they allow for additional learning about these future payoffs before a final decision on this launch is made and significant irreversible investments are undertaken. This is done in an environment where both, systematic market uncertainty and idiosyncratic technical risk affect the value of the project's commercial stage. The latter is driven by the investment decisions taken by the firm in the development stage of the project.

The R\&D investment literature started with Lucas (1971). He solved for the optimal allocation of effort throughout the development stage of the project in a general case where effort is controllable and time to completion is random. However, there is no modeling of learning and all uncertainty is private.

The next major work in the subject was done by Roberts and Weitzman (1981). In their model, the firm is continuously learning about they payoffs of a project as it invests through time . Based on a proportionality assumption about the learning process, they derived a diffusion process that the expected benefits of a project should follow. They do not take into account market uncertainty in their model. Consequently their 
results will be only applicable for a small set of projects where market uncertainty is negligible and could be ignored.

Grossman and Shapiro (1986) provided a few interesting models of R\&D programs under certainty and uncertainty in progress and time to completion. Again, the market dimension is absent and in their model of uncertainty in time to completion, the distribution of the time to completion is independent of actions taken by the firm and completely exogenous to the model.

McDonald and Siegel (1986) analyzed the value of waiting to invest. Their setting is useful when time to build is negligible when the development stage of the project has already been completed and a firm is considering whether to launch the commercial stage of a project or wait for more favorable market conditions.

Majid and Pyndick (1987) developed a continuous investment and time to build model. In their setting the only role of investment is to bring a project closer to completion. The learning dimension is excluded. Pyndick (1993) is probably the first to take into account market and technical uncertainty into a coherent framework. In this model revenues are fixed and costs are driven by market and technical uncertainty. However, his model does not make any distinction between the development and commercial stages of a project. This distinction turns out to be relevant as, in many industries, most of the learning takes place in the former.

More specific work, focused on particular industries or project characteristics, has followed. Messica and David (2000) analyzed the effect of the life cycle of revenues of the future project on the optimal investment allocation in the development stage. Cortazar et al. (2001) focused on optimal exploration investments in a mine under price and geological uncertainty. Bach and Paxson (2001) modeled investment in the drug development process. Schwarz and Soraya (2003), using a model similar to that of Pyndick (1993), analyzed investment in the IT industry both in acquisition and development projects.

Based on a few simplifying assumptions, we will set up a one period investment model to value R\&D investment opportunities in a context where both market and technical uncertainty are present. Technical learning will be modeled with a proportionality assumption for the relation between pilot investments and technical uncertainty reduction similar to that of Roberts and Weitzman. Since we will consider scenarios where market uncertainty is significant, our work will be based on a real options approach. This calls for a somewhat abstract but useful distinction between the value of claims to revenues and costs in the commercial stage, which will take the role of our underlying assets, and the value of the investment opportunity in the development stage, which will function as our option. Throughout our work we will use indistinctly the terms pilot, R\&D or development stage for the time period before the decision to launch the full scale project was 
made. For the stage that follows, we will use interchangeably the terms commercial or production stage. Our approach will be to account separately for technical and market uncertainty in our underlying assets. Spanning assumptions will be made on the market uncertainty of the project and technical uncertainty will be considered diversifiable and discounted at the risk free rate. Based on these assumptions, traditional financial engineering techniques will be appropriately applied to our setting, allowing us to obtain closed form solutions and comparative statics that will yield novel economic insights about the problem in question. Nevertheless, it is important to emphasize that our results can not be taken as strict non arbitrage prices of financial options but as plausible economic valuations of these real investment opportunities. Later on, most of our simplifying assumptions will be relaxed and the effects of this on our main results will be analyzed. However, in this paper we will choose to focus only on the one period investment assumption leaving a general continuous investment setting for future work.

The paper is structured as follows. Section 2 states our main assumptions and sets up the model. Section 3 values the opportunity to invest at a fixed investment level (what we call the second stage of the problem) and then solves for the optimal investment level and value of the investment opportunity. Section 4 has the main comparative statics and develops the economic insights implied by them. Section 5 analyzes the robustness of our results by relaxing some of the main assumptions and sketching the effects of these changes on the results obtained. Section 6 offers additional practical insight by linking the framework of this paper to common investment situations present in different industries and across the supply chain. Section 7 concludes.

\section{The Model}

In this section we provide the structure of the model that will be used throughout the paper. Our main assumptions will be stated and justified. They will provide us with a simple one period setting and closed form solutions for the value of our investment opportunity.

\subsection{General Structure of the Model}

The following assumptions will be used:

Assumption 1: The commercial stage of a project can only be launched after the pilot or development stage has been completed. Let $I$ be the total investment made on the pilot stage. For the pilot stage to be completed we need $I \in[\underline{I}, \bar{I}]$.

We can think of $\underline{I}$ as a feasibility bound. Below this level, it is not possible to go ahead with the commercial 
stage, since the minimum resources needed to guarantee an appropriate implementation, both on the technical and administrative side, have not been committed. On the other hand, $\bar{I}$ could be thought of as a budgetary bound. However, we could also think of it as an economic bound if, past this investment level, there is no gain from any additional expenditure made in the development stage. This is consistent with economic assumptions about decreasing returns of factors of production.

Assumption 2: Regardless of the level of investment $I$, the pilot or development stage takes a fixed time for completion which we will label T.

Assumption 3 At time 0, the decision of whether to invest or not in the pilot project is made. In the first case, the level of resources devoted to the pilot, I must also be decided. At time T, the decision to move ahead with the commercial stage or abandon the project must be also be taken.

These first assumptions, although somewhat restrictive, allow us to simplify the problem so that we can focus only on the one period tradeoff between the benefits of pilot investment, which are given by the increase in the value of the investment opportunity resulting from a higher resolution of technical uncertainty, and its cost. In Section 5, we consider the effect of relaxing these and some other assumptions that follow on our main results and conclusions. Nevertheless, throughout this paper, we restrict ourselves to the one period investment framework imposed by Assumption 3. By restricting ourselves to work on a one shot investment problem we lose the flexibility present in many real investment situations. However, this formulation allows us to obtain closed form solutions and comparative statics, and with them many important economic insights that would have been very difficult to obtain under a continuous or N-stage investment setting. Working in this direction, Errais and Sadowsky (2005) use Neuro Dynamic Programming techniques to value an Nstage pilot project in an infinite horizon setting in which investment drives the volatility of the processes followed by the underlying assets. However, the need to resort to approximate numerical solutions for the corresponding Bellman equation limits the extent to which we can learn about many of the issues that will be thoroughly analyzed in Section 4 of this paper.

\subsection{Assumptions about the Pilot Stage and Stochastic Processes}

The pilot stage will help reduce technical uncertainty with respect to the final costs of the project. In our setting, the revenues of the project will be only driven by market factors. The costs of the project will, in turn, be driven by both market and technical uncertainty. This is a reasonable assumption for most technical R\&D programs. However, we will see that our setting will also accommodate scenarios where the revenues of the project have also a significant idiosyncratic component. For example, a firm may want to decide the optimal funding that should be given to a marketing pilot project that will enhance its understanding of the 
different factors driving demand for a new product.

We could think of the total costs as the product of a price of inputs factor times an efficiency of inputs factor. Market considerations like the growth rate of the economy, the stock market, the unemployment level and other economic fundamentals will drive the price of inputs together with the entire revenue stream of the commercial project. The efficiency of inputs factor will be driven by idiosyncratic technical uncertainty, and it is here where the learning resulting from investment in the pilot stage raises the value of the option to invest.

Let $S_{t}$ be the value of a claim to all revenues of the commercial stage based on the information available at time $t$. Similarly, $K_{t}$, will be the value of a claim to all costs corresponding to the commercial stage, implementation and recurrent costs. We could think of $S_{t}$ and $K_{t}$ as the Net Present Value (NPV) of these cash flow streams assessed at time $t$. In addition, $S_{t}$ and $K_{t}$ will be the values of these claims, assuming that the commercial stage could be launched at the current time $t$. The information set upon which we condition includes all market and technical factors that influence the values of these claims. The assumption regarding the feasibility of the launch of the commercial stage is necessary, as it will allow us to formulate our problem in a Real Options framework by separating completely our underlying assets $\left(S_{t}\right.$ and $\left.K_{t}\right)$ and the option to invest. Even though at any time before completion $S_{t}$ and $K_{t}$ are not feasible claims, they will be so once the pilot stage is completed. Thus, this somewhat artificial definition, will not affect the results obtained.

Although some papers in the Real Options literature choose to use more basic variables as their underlying assets, such as output prices or demand, we have chosen to work with the NPVs of revenues and costs in a more general scenario, abstracting away from any specific properties of the project under consideration. Moreover, most pilot projects are inherently complex and their values are related to a few fundamental variables at the same time. Tracing their value back to these more fundamental variables may not be feasible in practice, or if so, may greatly affect the tractability of our setting.

We have chosen to model revenues and costs separately for two different reasons. First, market or technical uncertainty may not affect both revenues and costs, or if it does, it may not do so in the same way. For instance, in our setting, both types of uncertainty are relevant to costs, but revenues are only affected by market uncertainty. The second reason is that it is perfectly possible that the underlying commercial stage have a negative value, specially when it is technological and capital intensive. Keeping costs and revenues separately allows us to use log normality processes for each of them without restricting the value of the project to be positive all points in time.

Let the risk free rate in the economy be denoted by $r_{f}$. The following assumptions will also be made: 
Assumption 4: $S_{t}$ will follow a log normal process that is completely correlated with that of a tradable asset in the market. For simplicity, we will assume that this tradable asset is $S_{t}$ itself and that it follows the following diffusion process:

$$
d S_{t}=\alpha_{s} S_{t} d t+\sigma_{s} S_{t} d w_{1}
$$

Moreover, $S_{t}$ accrues dividends at rate $\delta_{s}$. This dividend rate $\delta_{s}$ has both a financial and economic interpretation. In strictly financial terms, it could be thought of as the yearly cash inflow that the project will currently generate once it were properly functioning. In this view $\delta_{s}$ is completely analogous to a dividend yield accrued by holding a stock. However, in a competitive market environment, a delay in launching a new product may have more undesirable consequences to a firm. Indeed, potential revenues lost due to market share gained by competitors, who may strike first while the firm is waiting for more favorable conditions, may be even more significant than the yearly revenue forgone. In settings like this, the economic opportunity cost of waiting should significantly increase the dividend yield $\delta_{s}$.

Assuming $S_{t}$ is tradable defines a uniquely market price of risk for $w_{1}$, which we will label $\lambda_{1}$. It is given by $\lambda_{1}=\left(\alpha_{s}+\delta_{s}-r_{f}\right) / \sigma_{s}$. The market price of risk is nothing else that the additional return in excess of the risk free rate that an investor requires per unit of volatility exposure to that particular source of risk.

This assumption is reasonable for big projects in industries where price and demand are very sensitive to economic fundamentals. This, in turn, depends among other factors on the nature of the industry, the product or service provided, and the dynamics of market competition. For example, few would argue that the revenues and variable costs resulting from investing in a pilot project to develop a new luxury car model are not correlated with the current or expected future state of the economy, or with the stock prices of the producing firm or of car manufacturing companies in general. In fact, the stock price of the firm already incorporates the assessment of the market about the success of this new car model. We have chosen a log normal process, since it is the most general and accepted way of modeling tradable market prices and many economic fundamentals. Nevertheless, depending on the time horizon, market characteristics or life cycle of revenues of the specific project under consideration, alternative processes that capture features like mean reversion and seasonality may need to be considered.

Assumption 5: $K_{t}$ will follow a log normal process that is driven by two Brownian Motions. The first one represents market uncertainty and is completely correlated with a tradable asset spanned by the market. Let us denote this tradable asset by $c_{t}$ and assume it follows: 


$$
d c_{t}=\alpha_{c} c_{t} d t+\sigma_{c} c_{t} d w_{2}
$$

with a dividend rate of $\delta_{c} . w^{2}$ is a Brownian motion representing the market uncertainty of costs. Moreover, the correlation between the costs market uncertainty with the revenues market uncertainty is $\rho$, i.e., $<$ $d w_{1}, d w_{2}>=\rho d t$. This uniquely specifies the market price of risk for $w_{2}$, which we will label $\lambda_{2}$ and it is equal to: $\lambda_{2}=\left(\alpha_{c}+\delta_{c}-r_{f}\right) / \sigma_{c}$.

A significant fraction of the costs of a project, specially in capital intensive industries, is driven by prices of materials and labor costs, which are, at worst, somewhat correlated with economic fundamentals. Hence, the assumption that cost market uncertainty is tradable is justified by the same arguments given in the previous assumption.

The second source of risk relevant for costs is a Brownian motion, which we will denote by $z_{t}$, representing technical uncertainty that is private to the firm. The technical volatility coefficient will depend on the level of investment made in the pilot stage $(I)$ in a way that we will make explicit shortly.

Thus, we assume that $K_{t}$ follows the following process:

$$
d K_{t}=\alpha_{k} K_{t} d t+\sigma_{k} K_{t} d w_{2}+\widehat{\sigma}_{z}(I) K_{t} d z
$$

where we have made explicit the dependence of the technical volatility on the level of investment in the development stage. The expected growth rate of costs, $\alpha_{k}$, is known by the firm and depends partly on the technological characteristics of the project and partly on the expected growth rate of relevant market factors such as labor costs or prices of inputs. In general, $\alpha_{k}$ could be dependent on the level of investment just as the technical volatility $\widehat{\sigma}_{z}(I)$ does. For ease of exposition, and since the main focus of our paper is to value of learning we choose to work with a constant growth rate in our main framework. In Section 5 we will consider relaxing these assumption to include an investment dependent growth rate. As we will see there this relaxation results in an additional effect on the value of the option to invest through changes in the effective risk neutral drift rate for costs. Unless these market factors are expected to growth significantly $\alpha_{k}$ will generally be negative as this technological investments are expected to increase efficiency, driving down costs on average.

Unlike market uncertainty, which is driven by the Brownian $w_{1}$ and $w_{2}$, technical risk is not spanned or tradable in the market. Thus, the market price of risk for technical uncertainty, $\lambda_{z}$, is not completely defined by non arbitrage considerations. As a consequence of the presence of technical uncertainty, we are left to work in a incomplete markets scenario and we need to resort to additional risk considerations in order 
to find a unique economic value for this investment opportunity. This is what Assumption 7 will do.

In the following assumption we will give some structure to the technical uncertainty and to $\widehat{\sigma}_{z}$, its diffusion term.

Assumption 6: Technical uncertainty resolved during the pilot stage increases with the level of investment. Hence, the maximum amount of technical uncertainty that could be reduced in the pilot stage, which we will call $\sigma_{z}$, corresponds to the maximum level of investment $\bar{I}$. Moreover, there is a proportionality relationship between investment and uncertainty reduction given by:

$$
\widehat{\sigma}_{z}(I)=\sigma_{z}\left(\frac{I}{\bar{I}}\right)^{\beta}
$$

This is a specific case of having $\widehat{\sigma}_{z}(I)=g(I)$ with $g(I)$ strictly increasing and continuous, $g(\bar{I})=\sigma_{Z}$ and $g(0)=0$. However, this proportional specification will give us, through the non negative parameter $\beta$ enough flexibility to model different forms of evolution of learning.

The parameter $\beta$ characterizes the marginal returns of pilot investment (where return in this context could be thought of as uncertainty reduction) and will be crucial for our problem. $\beta$ has a natural interpretation in this context. $0<\beta<1$ corresponds to decreasing returns to investment. If we invest a certain fraction of the maximum possible investment we will have resolved a higher fraction of the maximum technical uncertainty that could be resolved. This implies that the first dollar spent in the pilot stage is more productive in reducing technical uncertainty than the second one, which in turn is more productive than the third, and so on. Many projects will fall in this scenario for two reasons: first, it is related to general economic assumptions about decreasing marginal returns to capital and labor, and, in the research or development stage, the behavior of returns of capital and labor does not have to be fundamentally different to the one observed in the production process. The second reason is that in case the order of the independent experiments that are part of the pilot stage is not fixed, then the firm will want to put the projects with higher learning per dollar spent first. Decreasing returns to investment will be an obvious consequence of this reordering.

$\beta>1$ corresponds to increasing returns to investment. In this case, if we advance a certain fraction towards completion, the fraction of uncertainty reduced will be proportionally less. Later research dollars are more productive than earlier ones. This behavior is less prevalent than that of decreasing returns to investment. However, it also has some natural justifications, mainly based on learning curve type of arguments. Researchers and engineers get more productive in the course of time, especially when skills related to experiments in later stages are more relevant to skills required in earlier stages.

At this point the avid reader may have noticed that, although we mentioned reduction of technical uncertainty 
as one of the primary objectives of these pilot investments, the expression in (4) apparently implies the contrary, since the volatility term is increasing with the level of investment in the pilot stage. There is no such contradiction. Investment in the development stage will reduce the technical uncertainty that will be faced in the commercial stage after the full scale project is launched. As the total technical uncertainty faced by the project is fixed, more uncertainty resolved during the development stage, i.e., a higher $\widehat{\sigma}_{z}(I)$ will result in less exposure to technical risk at the launch of the project. Switching technical risk from the commercial to the development stage is beneficial for the firm, as risk in the development stage is resolved before undertaking the substantial and irreversible investments that are required to implement most projects in capital and technological intensive industries.

Assumption 7: Technical Uncertainty is completely diversifiable and, therefore, discounted at the risk free rate. In other words, the firm will not demand any additional return over the risk free rate for being exposed to this technical uncertainty. This will effectively "complete" the market by setting the market price of risk of $d z$ to zero. This assumption, widely used in the real options literature, may not apply in many real scenarios, where firms may actually place an important premium on risk. However, relaxing this assumption should not change our main results drastically, since as technical risk is shifted from the commercial stage to the pilot stage higher discounting in latter will compensate lower discounting in the former.

\section{Solution of the Model}

\subsection{Change of Measure}

To solve for the value of this investment opportunity and the optimal amount of resources that should be devoted to the pilot stage, we rely on Stochastic Calculus and standard Financial Engineering techniques. However, we should bear in mind that on standard financial settings, markets are complete, i.e., all different sources of risk are spanned by tradable assets in the economy. In this case, any financial derivatives whose payoff is constructed from the fundamental assets must have a unique price consistent with no arbitrage. Moreover, there is a unique risk neutral measure under which the price of any tradable asset discounted by the money market account is a martingale, i.e., the price of any tradable asset grows at the risk free rate.

In our scenario, the technical uncertainty faced by our projects is specific to the firm and not traded in the market. Hence, as in most real investment opportunities, we are working in an incomplete markets setting with infinitely many values consistent with the no arbitrage condition (in general these feasible values are defined by a lower bound and an upper arbitrage free bound derived by replication techniques) and infinitely many equivalent risk neutral measures that give us an arbitrage free economic valuation. Thus, to value 
these investment opportunities additional considerations as how the firm values private risk are needed. This is the role of Assumption 7. By adding the additional risk consideration that the firm takes technical risk as completely diversifiable, it allows us to work with a unique risk neutral measure. Hence, unlike in complete market scenarios, the values obtained for these real investment opportunities shall be regarded as plausible economic valuations and not as strict non arbitrage prices.

All technical details relevant to the construction of the risk neutral measure $Q$ will be left for the appendix. It follows from the appendix that the processes that $S_{t}$ and $K_{t}$ follow under $Q$ are given by:

$$
\begin{aligned}
d S_{t} & =\left(r_{f}-\delta_{s}\right) S_{t} d t+\sigma_{s} S_{t} d w_{1}^{Q} \\
d K_{t} & =\left(r_{f}-\delta_{k}\right) K_{t} d t+\sigma_{k} K_{t} d w_{2}^{Q}+\sigma_{z}(I / \bar{I})^{\beta} K_{t} d z^{Q}
\end{aligned}
$$

with $w_{1}^{Q}, w_{2}^{Q}$ and $z^{Q}$ being standard Brownian motions under $Q$. Moreover $w_{1}^{Q}$ and $w_{2}^{Q}$ have a correlation of $\rho$ and $z^{Q}$ is independent of both $w_{1}^{Q}$ and $w_{Q}^{2}$. $\delta_{k}$ is a constant that plays a role of a "dividend rate" for costs and represents nothing more than the difference between the risk adjusted return for costs and its intrinsic growth rate.

$$
\delta_{K}=r_{f}-\alpha_{k}+\frac{\alpha_{c}+\delta_{c}-r}{\sigma_{c}} \sigma_{k}
$$

We will work hereafter in the risk neutral measure $Q$. Thus, for notational simplicity, from now on we will omit the superscripts $Q$ from our Brownian motions.

\subsection{Second Stage: Value of the Option given an Investment Level}

We are now ready to solve our problem. For convenience, instead of choosing the total investment level $I$ from $[\underline{I}, \bar{I}]$ we will choose the $\alpha=I / \bar{I}$, the fraction of the maximum pilot investment possible, where $\alpha \in\left[\alpha_{0}, 1\right]$, being $\alpha_{0}=\underline{I} / \bar{I}$ the minimum fraction feasible.

The problem of valuing the option to invest at a fix investment level $\alpha$ results to be nothing more than pricing an option to exchange one asset for another, with the amount invested in the pilot stage driving the volatility of one of the assets.

The following proposition gives the value of the investment opportunity corresponding to an investment fraction of $\alpha$.

Proposition 3.1 Under Assumptions 1-7, when a fraction $\alpha \in\left[\alpha_{0}, 1\right]$ is invested in the pilot stage, the value of the investment opportunity $V(\alpha)$, is given by: 


$$
V(\alpha)=S_{0} e^{-\delta_{s} T} N\left(d_{1}(\alpha)\right)-K_{0} e^{-\delta_{k} T} N\left(d_{2}(\alpha)\right)-\alpha \bar{I}
$$

where $\delta_{k}$ is given by $(7)$ and $d_{1}(\alpha)$ and $d_{2}(\alpha)$ are given by:

$$
\begin{array}{r}
d_{1}(\alpha)=\frac{\ln \left(S_{0} / K_{0}\right)+\left(\delta_{k}-\delta_{S}+\widehat{\sigma}^{2}(\alpha) / 2\right) T}{\widehat{\sigma}(\alpha) \sqrt{T}} \\
d_{2}(\alpha)=d_{1}(\alpha)-\widehat{\sigma}(\alpha) \sqrt{T} \\
\widehat{\sigma}(\alpha)=\sqrt{\sigma_{s}^{2}-2 \rho \sigma_{s} \sigma_{k}+\sigma_{k}^{2}+\sigma_{z}^{2} \alpha^{2 \beta}}
\end{array}
$$

Proof: First note that after the firm decides to invest a level $\alpha \bar{I}$ it obtains nothing more than the option to exchange one asset (total revenues) for another (total costs) at the time of completion of the pilot stage.

The value of this option will be obtained by working in the risk neutral measure $Q$ constructed in the appendix. Note that under $Q, S_{t}$ and $K_{t}$ follow (5) and (6) respectively and the final payoff at time $\mathrm{T}$ that determines the boundary condition is given by: $\max \left[S_{T}-K_{T}, 0\right]$.

The value of an option to exchange one asset for another was originally derived by Margrabe (1978) who solved the non-arbitrage PDE that the value of this option has to follow. However, the solution could be easily obtained if we do a further change of measure and work in a risk neutral forward with respect to the portfolio of $K_{t}$ and its dividends. Both procedures result in (8). See Appendix for details on the calculation of this expression as well as that of equation (21).

We must also note that solution obtained is independent on the risk free rate and only the dividend rates of revenues and costs matter at the end. We could also think of the solution as being the value of a European Call with a fixed strike $K=K_{0}$, a risk free rate of $\delta_{k}$ and a diffusion term of revenues $\sigma_{s}=\widehat{\sigma}(\alpha)$.

Let's now characterize the sensitivities of the option value to the effective volatility term $\widehat{\sigma}(\alpha)$ and the fraction of investment $\alpha$.

Borrowing some terms from the financial derivatives literature we will define vega $(\widehat{\sigma})=\delta V(\widehat{\sigma}) / \delta \widehat{\sigma}$ and $\operatorname{vomma}(\widehat{\sigma})=\delta^{2} V(\widehat{\sigma}) / \delta \widehat{\sigma}^{2}$.

We can now characterize the sensitivity of the option value to the investment level.

Proposition 3.2 Let's denote $V_{\alpha}$ and $V_{\alpha \alpha}$ the partial derivatives of the value of the investment opportunity with respect to the fraction of investment. Then the following equalities hold: 


$$
\begin{array}{r}
\operatorname{vega}(\widehat{\sigma}(\alpha))=S_{0} e^{-\delta_{S} T} N^{\prime}\left(d_{1}(\alpha)\right) \sqrt{T} \\
\operatorname{vomma}(\widehat{\sigma}(\alpha))=\frac{\operatorname{vega}(\widehat{\sigma}(\alpha)) d_{1}(\alpha) d_{2}(\alpha)}{\widehat{\sigma}(\alpha)} \\
V_{\alpha}(\alpha)=\operatorname{vega}(\widehat{\sigma}(\alpha)) \beta \frac{\sigma_{Z}^{2}}{\widehat{\sigma}(\alpha)} \alpha^{2 \beta-1}-\bar{I}=S_{0} e^{-\delta_{S} T} N^{\prime}\left(d_{1}(\alpha)\right) \sqrt{T} \beta \frac{\sigma_{Z}^{2}}{\widehat{\sigma}(\alpha)} \alpha^{2 \beta-1}-\bar{I} \\
V_{\alpha \alpha}(\alpha)=\operatorname{vega}(\widehat{\sigma}(\alpha)) \beta \frac{\sigma_{Z}^{2}}{\widehat{\sigma}(\alpha)} \alpha^{2 \beta-2}\left[\beta \frac{\sigma_{Z}^{2}}{\widehat{\sigma}^{2}(\alpha)} \alpha^{2 \beta}\left(d_{1}(\alpha) d_{2}(\alpha)-1\right)+(2 \beta-1)\right]
\end{array}
$$

where $d_{1}(\alpha), d_{2}(\alpha)$ and $\widehat{\sigma}(\alpha)$ are given by (9), (10) and (11) respectively.

We could break up equation (14) into two components the marginal revenues, given by the first term in the equation and marginal costs which are constant and equal to $\bar{I}$.

\subsection{Solution of The First Stage: Finding the Optimal Investment Level}

At time 0 , the firm chooses an investment fraction $\alpha \in\{0\} \cup\left[\alpha_{0}, 1\right]$ to maximize:

$$
\max _{\alpha \in\{0\} \cup\left[\alpha_{0}, 1\right]} V(\alpha)-\alpha \bar{I}
$$

where $V(\alpha)$ is given by (8) for $\alpha \in\left[\alpha_{0}, 1\right]$ and $V(0)=0$, with the latter corresponding to abandoning the project. We will call the optimal investment fraction $\alpha^{\star}$ and the value of the investment opportunity $V^{\star}=V\left(\alpha^{\star}\right)$.

Before solving the problem and analyzing its comparative statics we will make the following simplifications. First, we can assume without loss of generality that $\bar{I}=1$, since we can scale all the terms in (16) by $\bar{I}$. Then, let $\sigma_{m}^{2}=\sigma_{s}^{2}-2 \rho \sigma_{s} \sigma_{k}+\sigma_{k}^{2}$ and $\kappa=\sigma_{z}^{2} / \sigma_{m}^{2}$, so that $\widehat{\sigma}(\alpha)$, which we will call the effective volatility term is equal to $\sigma_{m} \sqrt{1+\kappa \alpha^{2 \beta}}$. $\sigma_{m}$ corresponds to the total tradable uncertainty of the project, which for this setting is also the total market uncertainty. Note that this market uncertainty is higher when revenues and costs are negatively correlated (positive shocks in revenues are reinforced with decreases in costs and viceversa) and is lower when they are positively correlated (increases in revenues are somewhat compensated by increases in cost). The ratio $\kappa$ will play a very important role in the solution to the problem. It is a measure of the relative importance of the technical uncertainty with respect to the market uncertainty of the project and it will depend on a series of factors like whether the final product is basic or luxury, the dynamics of competition, existence of possible substitutes or how capital intensive the project is. Thus $\kappa$ will vary according to the industry in general, and to the specific project in particular. For instance, this 
ratio will generally be higher in the pharmaceutical or food industries and lower in the auto industry or the high tech sector.

Let us denote as $M R(\alpha)$ the first term of $V_{\alpha}$ in (14). The behavior of this marginal revenue function will turn out to be crucial for our results and economic analysis, so we need to characterize completely. If we fixed $\sigma_{m}$ and $\kappa$ then from (14) our marginal revenue function $M R(\alpha)$ becomes:

$$
M R(\alpha)=S_{0} e^{-\delta_{S} T} N^{\prime}\left(d_{1}(\alpha)\right) \sqrt{T} \beta \frac{\kappa \sigma_{m}}{\sqrt{1+\kappa \alpha^{2 \beta}}} \alpha^{2 \beta-1}
$$

Proposition 3.3 The marginal revenue function is strictly positive for any $\alpha>0$. If $\beta \leq 0.5$ and $d_{1}(0) d_{2}(0)<1+(1-2 \beta)(1+\kappa) /(\beta \kappa)$ then $M R(\alpha)$ is strictly decreasing in $\alpha$ for $\alpha \in[0,1]$. If $\beta>0.5$ then there exists a unique threshold $\widetilde{\alpha}$ such that for any $0<\alpha<\widetilde{\alpha}$ the marginal revenue function is strictly increasing in $\alpha$ and for any $\alpha>\widetilde{\alpha}$ the marginal revenue function is strictly decreasing in $\alpha$. Moreover, when $\widetilde{\alpha} \in[0,1]$ then $\delta \widetilde{\alpha} / \delta \beta>0$.

Proof: Take the case of $\beta \leq 0.5$. We will call this scenario strong decreasing returns to investment. First note that the assumption $d_{1}(0) d_{2}(0)<1+(1-2 \beta)(1+\kappa) /(\beta \kappa)$ is used to rule out pathological cases of low volatility and extreme moneyness ${ }^{1}$. It is equivalent to saying that $\sigma_{m}^{4} T^{2} / 4+\sigma_{m}^{2}(1+(1-2 \beta)(1+\kappa) /(\beta \kappa)) T-$ $\left[\ln \left(S_{0} / K_{0}\right)+\left(\delta_{k}-\delta_{s}\right) T\right]^{2}>0$ and, as said before, it will not hold in cases of extremely low volatility and when it is very likely that either the commercial stage would be launched or that it would discarded at time T. As we are interested in modelling situations where uncertainty and optionality play a significant role such scenarios will not economically relevant for our analysis.

We can easily check that $d_{1}(\alpha) d_{2}(\alpha)$ is increasing in $\alpha$ and $\left(1+\kappa \alpha^{2 \beta}\right) /\left(\kappa \alpha^{2 \beta}\right)$ is decreasing in $\alpha$. This together with our assumption implies that for any $\alpha \in[0,1]$ :

$$
d_{1}(\alpha) d_{2}(\alpha)<\frac{(1-2 \beta)\left(1+\kappa \alpha^{2 \beta}\right)}{\beta \kappa \alpha^{2 \beta}}+1
$$

This results in the term inside brackets in (15) being negative and $M R(\alpha)$ being strictly decreasing in $\alpha \in[0,1]$. At $\alpha=0, M R$ is unbounded for any $\beta<0.5$, i.e., $\lim _{\alpha \rightarrow 0} M R(\alpha)=+\infty$, and it is equal to a positive number for $\beta=0.5$. Moreover as $\alpha$ goes to infinity, $M R(\alpha)$ goes to zero. This results in $V(\alpha)$ being a strictly increasing concave function and, therefore, there exists the possibility of an interior solution to our problem. The restrictions on $\alpha \in\left[\alpha_{0}, 1\right]$ and some feasible combinations of the other parameters

\footnotetext{
${ }^{1}$ We define moneyness as the ratio $\frac{S_{0}}{K_{0} e^{\left(\delta_{k}-\delta_{s}\right) T}}$. A high level of moneyness implies that the commercial stage will be very likely to be launched in the future, while a low level of moneyness implies that the commercial stage will most likely be discarded. In most of the financial literature this term is defined as $S_{0} / K_{0}$, here we introduce an additional correction factor to take the drift effect into account.
} 
may also yield solutions on the boundary of $\alpha$, i.e., $\alpha=\alpha_{0}$ or $\alpha=1$. Decreasing marginal revenues in the fraction of investment is consistent with the fact that $\beta \leq 0.5$ characterizes strong decreasing returns to investment, each additional dollar spent in the pilot stage is a lot less productive in reducing uncertainty than the previous one, in other words, the more investment that has been made along the way, the less the marginal payoff derived from additional investment.

Now, let's analyze the case when $\beta>0.5$. In this case $M R(0)=0$ and $M R(\alpha)>0$ for any positive $\alpha$. From (17) and the fact that as $\alpha$ goes to infinity, $\widehat{\sigma}(\alpha)$ and $d_{1}(\alpha)$ do so as well we have that $\lim _{\alpha \rightarrow+\infty} M R(\alpha)=0$. Moreover, since the term inside the brackets in equation (15) is positive for $\alpha$ small enough and negative for large enough $\alpha$, we can guarantee the existence of a positive $\widetilde{\alpha}$ such that $V_{\alpha \alpha}=0$. This $\widetilde{\alpha}$ is the solution to the following equation:

$$
\frac{2 \beta-1}{\beta \kappa}=\frac{\alpha^{2 \beta}\left(1-d_{1}(\alpha) d_{2}(\alpha)\right)}{1+\kappa \alpha^{2 \beta}}
$$

Now, since $d_{1}(\alpha) d_{2}(\alpha)$ is decreasing in $\alpha$ and $\alpha^{2 \beta} /\left(1+\kappa \alpha^{2 \beta}\right)$ is increasing in $\alpha$, the RHS of (18) is increasing in $\alpha$. This guarantees the uniqueness of $\widetilde{\alpha}$. Hence, $M R(\alpha)$ is strictly increasing $(V(\alpha)$ is strictly increasing and convex) for any $0<\alpha<\widetilde{\alpha}$ and strictly decreasing $(V(\alpha)$ is strictly increasing and concave) for $\alpha>\widetilde{\alpha}$. Typical shapes of the marginal revenue function for $\beta<0.5, \beta \in[0.5,1]$ and $\beta>1$ are plotted in figure 1 .

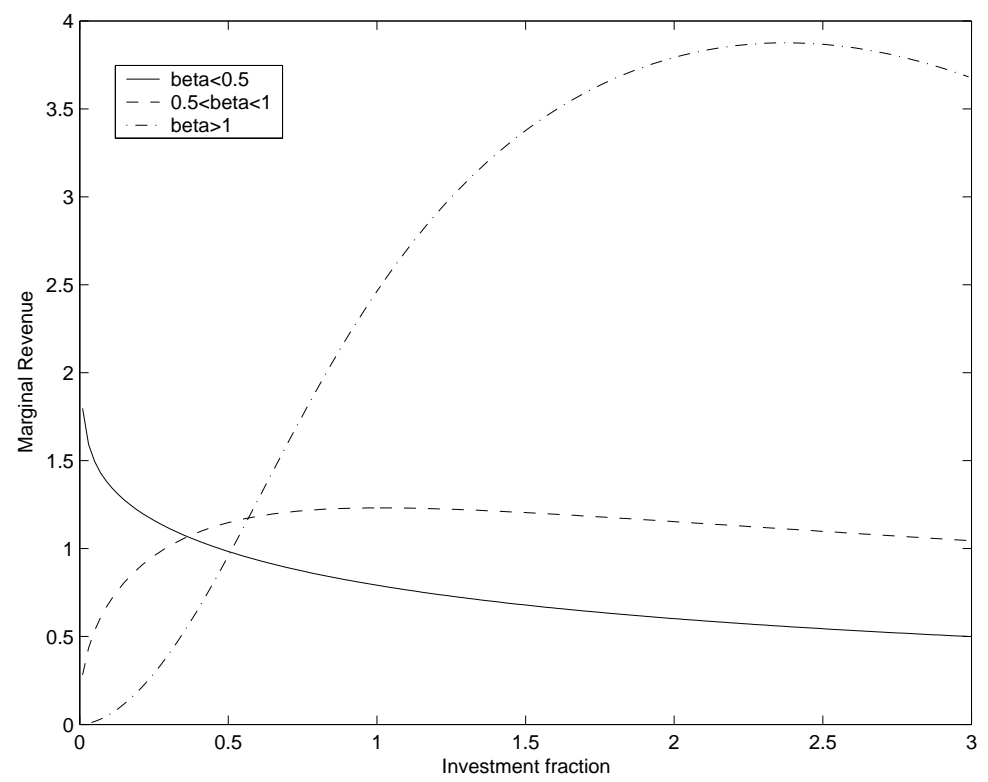

Figure 1: Typical shapes of the Marginal Revenue function for $\beta<0.5,0.5<\beta<1$ and $\beta>1$ as a function of level of investment $\alpha$

In order to show that if $\widetilde{\alpha} \in[0,1]$ then $\delta \widetilde{\alpha}(\beta) / \delta \beta>0$, we differentiate implicitly (18) with respect to $\beta$ to 


$$
\begin{gathered}
\frac{1}{\beta^{2} \kappa}=\frac{\delta}{\delta \alpha}\left[\frac{\alpha^{2 \beta}\left(1-d_{1}(\alpha) d_{2}(\alpha)\right)}{1+\kappa \alpha^{2 \beta}}\right] \frac{\delta \alpha}{\delta \beta}+\frac{\delta}{\delta \beta}\left[\frac{\alpha^{2 \beta}\left(1-d_{1}(\alpha) d_{2}(\alpha)\right)}{1+\kappa \alpha^{2 \beta}}\right] \\
\frac{\delta \alpha}{\delta \beta}=\left\{\frac{\delta}{\delta \alpha}\left[\frac{\alpha^{2 \beta}\left(1-d_{1}(\alpha) d_{2}(\alpha)\right)}{1+\kappa \alpha^{2 \beta}}\right]\right\}^{-1}\left\{\frac{1}{\beta^{2} \kappa}-\frac{\delta}{\delta \beta}\left[\frac{\alpha^{2 \beta}\left(1-d_{1}(\alpha) d_{2}(\alpha)\right)}{1+\kappa \alpha^{2 \beta}}\right]\right\}
\end{gathered}
$$

For $\alpha \in[0,1]$ the effective volatility term $\widehat{\sigma}(\alpha)$ is decreasing in $\beta$. Thus, $d_{1}(\alpha) d_{2}(\alpha)$ is increasing in $\beta$. The term $\left(\alpha^{2 \beta}\right) /\left(1+\kappa \alpha^{2 \beta}\right)$ is decreasing in $\beta$ for $\alpha \in[0,1]$. All this implies that the R.H.S of (18) is decreasing in $\beta$. This together with the previous expression and the fact that the R.H.S of (18) is increasing in $\alpha$ imply that $\delta \widetilde{\alpha} / \delta \beta<0$ completing the proof.

The fact that the inflection point of the marginal revenue functions is increasing in $\beta$ implies that as we move from decreasing to increasing returns to investment it is more likely that the region in which $M R(\alpha)$ is decreasing will fall outside of our feasible range, $\left[\alpha_{0}, 1\right]$. For $\beta$ high enough we have that $\widetilde{\alpha}>1$ and we will only be faced with an increasing $M R(\alpha)$ in our region of interest. In fact, we will see that for the case of increasing returns to investment, $\beta>1$, this will happen for all reasonable combination of parameters. The opposite occurs as $\beta$ approaches 0.5 . In this case, $\widetilde{\alpha}$ keeps on decreasing until $\widetilde{\alpha}<\alpha_{0}$ and we are left with a decreasing $M R$ function in our region of interest as our original intuition would suggest. Additional insights about how the learning parameter affect the value of our investment opportunity and optimal investment decision will be given in the following section.

Note also that even though we have characterized $M R(\alpha)$ for any $\alpha>0$, for the solution of (16) we will only be interested in the behavior of this function on our feasibility region $\left[\alpha_{0}, 1\right]$.

Let $\widehat{\alpha}$ denote the solution to the equation $M R(\alpha)=1$ when it exists, i.e, $\widehat{\alpha}$ solves:

$$
S_{0} e^{-\delta_{S} T} N^{\prime}\left(d_{1}\right) \sqrt{T} \beta \frac{\kappa \sigma_{m}}{\sqrt{1+\kappa \alpha^{2 \beta}}} \alpha^{2 \beta-1}=1
$$

When $\beta>0.5$, due to the inflection point of $V(\alpha), \widehat{\alpha}$ may have either two or no solutions. For the former case we will denote them as $\widehat{\alpha}_{L}$ and $\widehat{\alpha}_{H}$ with $\widehat{\alpha}_{L}<\widetilde{\alpha}<\widehat{\alpha}_{H}$.

Now we are ready for a complete characterization of the solution to our investment problem, which is stated in the following Proposition.

Proposition 3.4 Let $\widetilde{\alpha}$ and $\widehat{\alpha}$ be defined as the solutions to (18) and (19) when they exist. Then the solution to our investment problem (16), which we will denote as $\alpha^{\star}$ is given by: 
a) For $0<\beta \leq 0.5$. We have 3 subcases:

a1) If $M R\left(\alpha_{0}\right)>1$ and $\alpha_{0}<\widehat{\alpha}<1$ then $\alpha^{\star}=\widehat{\alpha}$.

a2) If $M R\left(\alpha_{0}\right)>1$ and $\widehat{\alpha}>1$ then $\alpha^{\star}=1$.

a3) If $M R\left(\alpha_{0}\right)<1$ then $\alpha^{\star}=\arg \max _{\alpha \in\left\{0, \alpha_{0}\right\}} V(\alpha)$.

b) For $\beta>0.5$. We have 4 subcases:

b1) There are no solutions to (19) in $\left[\alpha_{0}, 1\right]$ with $M R(\alpha)<1$ for all $\alpha \in\left[\alpha_{0}, 1\right]$, then $\alpha^{\star}=\arg \max _{\alpha \in\left\{0, \alpha_{0}\right\}} V(\alpha)$.

b2) There are no solutions to (19) in $\left[\alpha_{0}, 1\right]$ with $M R(\alpha)>1$ for all $\alpha \in\left[\alpha_{0}, 1\right]$, then $\alpha^{\star}=\arg \max _{\alpha \in\{0,1\}} V(\alpha)$.

b2) The only solution to (19) in $\left[\alpha_{0}, 1\right]$ is $\widehat{\alpha}_{L}$. Then $\alpha^{\star}=\arg \max _{\alpha \in\left\{0, \alpha_{0}, 1\right\}} V(\alpha)$. This scenario could only occur when $\widetilde{\alpha}>\alpha_{0}$.

b3) The only solution to (19) in $\left[\alpha_{0}, 1\right]$ is $\widehat{\alpha}_{H}$. Then $\alpha^{\star}=\arg \max _{\alpha \in\left\{0, \widehat{\alpha}_{H}\right\}} V(\alpha)$. This scenario could only take place if $\widetilde{\alpha}<1$.

b4) There are two solutions, to (19) in $\left[\alpha_{0}, 1\right]$. Then $\alpha^{\star}=\arg \max _{\alpha \in\left\{0, \alpha_{0}, \widehat{\alpha}_{H}\right\}} V(\alpha)$. This scenario could only occur when $\alpha_{0}<\widetilde{\alpha}<1$.

Proof: Follows trivially by simple analysis and the characterization of $M R(\alpha)$ given before.

The general characterization of the solution given above may be somewhat overwhelming given all the possible combinations involved. However, we will see that for most reasonable combination of parameters all this set of possible combinations could be dramatically reduced and some general conclusions may be obtained. Moreover, as to focus exclusively on the effect of technical uncertainty reduction and the returns to investment in the optimal investment decision we will focus on the case where $V(\alpha)>\alpha$ for $\alpha \in\left[\alpha_{0}, 1\right]$, i.e, all the feasible levels of investment are worth undertaking. This will get rid of the no investment solutions to our problem, so that the solution will rely entirely on our $M R(\alpha)$ function and how it is affected by our different parameters of interest.

Figure 2 shows two typical situations where an interior point solution exist for $\beta \leq 0.5$ and $\beta>0.5$, which correspond to the cases a1 and either b3 or b4 (depending on the value of $\alpha_{0}$ ) respectively.

For the case of strong decreasing returns to investment $0<\beta \leq 0.5$ an interior solution exists if marginal revenues start above one at $\alpha_{0}$, the beginning of the feasible region, and end below one at the end of the feasible region $\alpha=1$ (case a1). An existence of an interior point solution is also possible for $\beta>0.5$, (cases b3 and b4) and the closer $\beta$ is to 0.5 , the greater combination of parameters for which this will occur. With $\beta>0.5$ the marginal revenues are first increasing and then decreasing. For this case, the lower $\beta$ the more learning we get from the first research dollars invested with respect to later investment. This results 


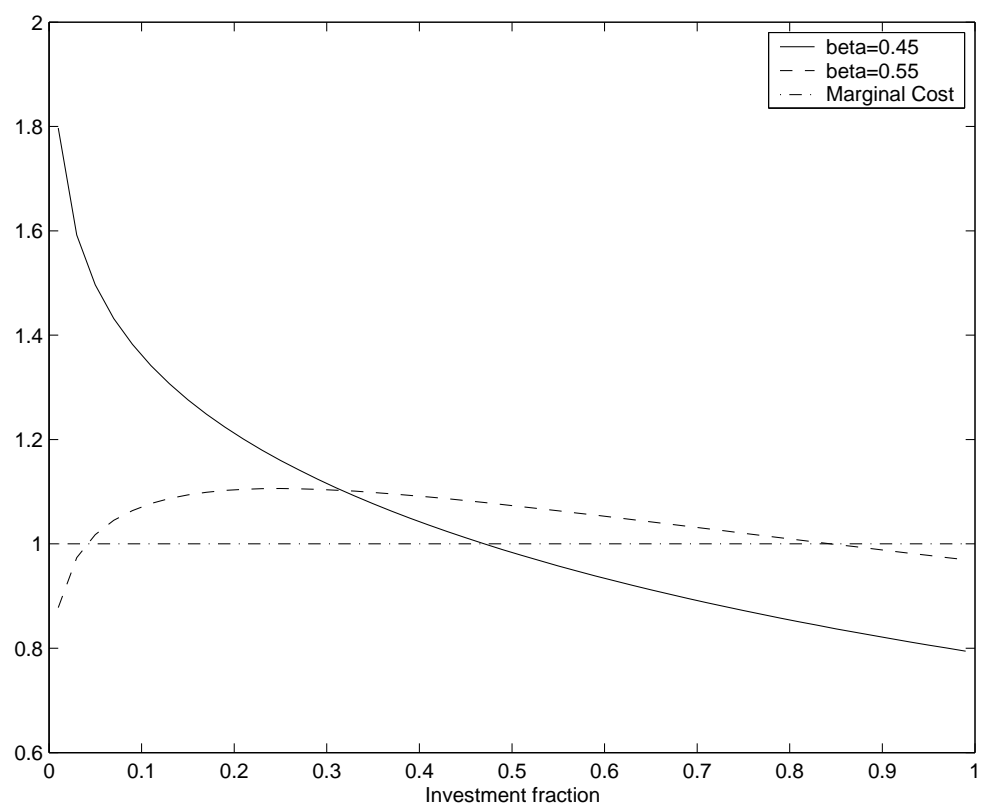

Figure 2: Illustration of the existence of an interior point solution, cases a1 $(\beta \leq 0.5)$ and either b3 or b4 $(\beta>0.5)$ respectively

in a lower inflection point for the marginal revenue function and hence, in a greater interval on which the marginal revenues are decreasing in the fraction of investment $\alpha$. We will be especially interested in the case of an interior solution, since the optimal investment fraction and the value of the option will change smoothly with changes in any of our parameters of interest. It is in these cases, where we will get most of the interesting comparative statics and economic insights of our model.

Following the opposite reasoning as $\beta$ increases away from 0.5 , the higher the share of the total learning for which later research dollars invested are responsible, the higher the inflection point of the marginal revenue function, and the bigger the set of parameter combinations for which marginal revenues are increasing in our feasible range $(\widetilde{\alpha}>1)$. The following expression is necessary and sufficient for this condition to hold:

$$
d_{1}(1) d_{2}(1)=\frac{\left(\ln \left(S_{0} / K_{0}\right)+\left(\delta_{k}-\delta_{s}\right) T\right)^{2}-\widehat{\sigma}^{4}(1) T^{2} / 4}{\widehat{\sigma}^{2}(1) T}>1-\frac{2 \beta-1}{\beta} \frac{\sqrt{1+\kappa}}{\kappa}
$$

In fact, we can easily check that for increasing returns to investment $(\beta>1)$ this inequality is satisfied for most, if not all, reasonable parameter combinations. When this occurs the optimal investment level will lie on the boundary points: either the minimum investment required for a feasible implementation will be made $\left(\alpha^{\star}=\alpha_{0}\right)$ or the maximum possible investment will be undertaken $\left(\alpha^{\star}=1\right)$. In this case, the optimal investment decision will not change smoothly with changes in the key parameters of our models. For this reason and the fact that $\beta<1$ corresponds to more realistic specifications of the learning process, this case 
will not be as interesting to our analysis as the case when interior solutions take place.

\section{Economic Analysis and Main Comparative Statics}

In this section we will go over the most important comparative statics and economic insights that follow from our model.

First, and not surprisingly, the solution to our problem will be very sensitive to the learning coefficient $\beta$. Since the focus of our paper is to value learning, this parameter is key to our model, since it affects the way learning is distributed across the cumulative investment path.

The parameters that affect the volatility of our option, $\kappa=\sigma_{z}^{2} / \sigma_{m}^{2}$ (the relative weight of technical and market uncertainty in our project) and the absolute volatility terms $\sigma_{m}$ and $\sigma_{z}$ will also be of economic significance, as market and technical uncertainty have different effects in the incentive to invest.

The initial assessments of revenues and $\operatorname{costs} S_{0}$ and $K_{0}$ and the drift parameters $\delta_{s}$ and $\delta_{k}$ are also relevant since they affect the moneyness of our option and, through it, the marginal gain in its value resulting from an increase in uncertainty resolved.

We will explore the effect of changes of all of this parameters in our result and try to obtain general economic insights that we can extrapolate to many investment situations. Since some of these calculations are algebraically intensive, the readers are referred to the appendix for the complete calculations and all other details left out in this section.

\subsection{Sensitivity to the Learning Coefficient $(\beta)$}

The learning coefficient $\beta$ characterizes "returns" to investment in the pilot stage, where we could think of returns as the total technical uncertainty resolved. We saw in the previous that this coefficient is the main driver of the shape of the marginal revenue function. As expected, low values of $\beta$ (higher contribution to total learning from the first dollars invested) will result in a decreasing marginal revenue function in the feasible range for $\alpha$. On the other hand, high values of $\beta$ (more contribution to total learning from later research dollars invested) result, in general, in an increasing marginal revenue function along the feasible investment range.

It is also worthwhile to emphasize that when the maximum possible investment is made $(\alpha=1)$ the total uncertainty reduction is the same regardless of the value of $\beta$. For this case $\beta$ only affects the way in which learning is distributed across the cumulative investment path of the pilot project.

Using simple principles of monotone comparative statics, we can see that the effect of $\beta$ on the optimal 
investment level is governed by the behavior of the function $\delta M R(\alpha) / \delta \beta$.

Proposition 4.1 For a fix $\beta$ there exists an $\breve{\alpha}(\beta) \in(0,1)$ such that $\delta M R(\alpha) / \delta \beta<0$ for any $\alpha \in[0, \breve{\alpha}(\beta))$ and $\delta M R(\alpha) / \delta \beta>0$ for any $\alpha \in(\breve{\alpha}(\beta), 1]$.

Proof Since the proof of this proposition is mainly algebraic and has little economic relevance, we will leave it for the appendix.

The result has a nice economic interpretation. Remember that $\beta$ governs the way in which learning relates to total investment. A higher $\beta$ implies that the later dollars invested are responsible for a higher share of the cumulative learning with respect to earlier dollars invested. As $\beta$ is increased, more learning is shifted from the beginning of the cumulative investment path (low $\alpha$ ) to its end (high $\alpha$ ). This causes a decrease in marginal revenues for low $\alpha$ and an increase in marginal revenues for high $\alpha$, since now later marginal investments are resolving more additional uncertainty. This property will help us characterize the sensitivity of the option value and the optimal investment fraction to $\beta$.

Proposition 4.2 The option value $V^{\star}$ is decreasing in $\beta$. If the optimal investment decision $\alpha^{\star}(\beta)$ is at at an interior point $\left(\alpha^{\star}(\beta) \in\left[\alpha_{0}, 1\right]\right)$ then an increase in $\beta$ raises optimal investment if $\alpha^{\star}(\beta)>\breve{\alpha}(\beta)$ and decreases the level of investment otherwise. The first case is more common, while the second one occurs only for low feasible thresholds $\alpha_{0}$ and very high levels of market uncertainty.

Proof: If the current optimal investment decision is at an interior point then it must be the case that $M R\left(\alpha^{\star}(\beta), \beta\right)=1$. Moreover $M R(\alpha)$ must be decreasing in a neighborhood of $\alpha^{\star}$, otherwise we would have a local minimum. Assume an infinitesimal increase from $\beta$ to $\beta^{\prime}>\beta$ occurs. If $\alpha^{\star}(\beta)>\breve{\alpha}(\beta)$ then by proposition 4.1, $M R\left(\alpha^{\star}(\beta), \beta^{\prime}\right)>1$. Then the current investment level can not be optimal, since we can increase the value of the option by increasing $\alpha^{\star}$ until $M R\left(\alpha^{\star}, \beta^{\prime}\right)=1$. Hence, $\alpha^{\star}\left(\beta^{\prime}\right)>\alpha^{\star}(\beta)$. Figure 3 illustrate this reasoning. The same reasoning holds for an infinitesimal decrease in $\beta$ and for the case where $\alpha^{\star}(\beta)<\breve{\alpha}(\beta)$.

Now let the value of the option be $V^{\star}=V\left(\alpha^{\star}(\beta), \beta\right)$, where we are writing explicitly the dependence on $\alpha$ and $\beta$, our parameters of interest. Taking the derivative with respect to $\beta$ :

$$
\frac{\delta V^{\star}}{\delta \beta}=\left.\frac{\delta V}{\delta \alpha}\right|_{\left(\alpha^{\star}(\beta), \beta\right)} \frac{\delta \alpha^{\star}(\beta)}{\delta \beta}+\left.\frac{\delta V}{\delta \beta}\right|_{\left(\alpha^{\star}(\beta), \beta\right)}
$$

If $\alpha^{\star}(\beta)$ is an interior point then by the envelope condition $\left.\frac{\delta V}{\delta \alpha}\right|_{\left(\alpha^{\star}(\beta), \beta\right)}=0$ and since

$$
\frac{\delta V}{\delta \beta}=\frac{S_{0} e^{-\delta_{S} T} N^{\prime}\left(d_{1}\right) \alpha^{2 \beta} \sigma_{m} \ln (\alpha)}{\sqrt{1+\kappa \alpha^{2 \beta}}}<0
$$




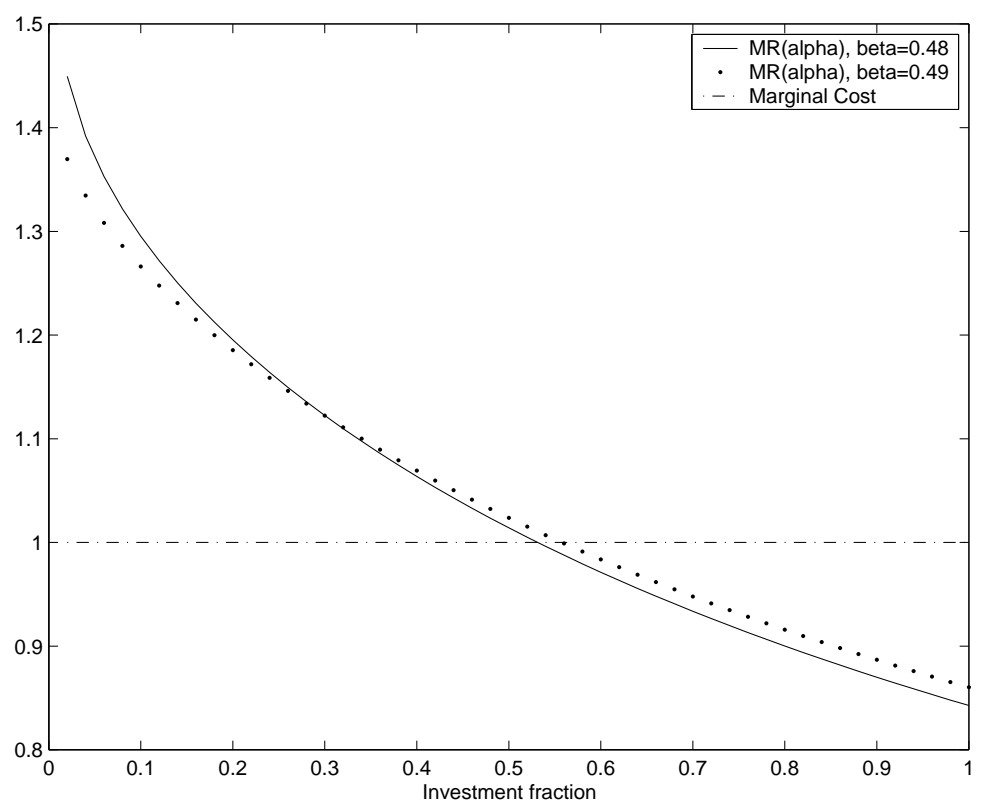

Figure 3: Change on the optimal investment fraction as a result of an increase in $\beta$ for the case of an interior point solution and $\alpha^{\star}(\beta)>\breve{\alpha}(\beta)$

then we have that the option value is decreasing in $\beta$.

Now, in case the optimal decision is at a boundary point, then the envelope condition $\left.\frac{\delta V}{\delta \alpha}\right|_{\alpha^{\star}(\beta)}=0$ does not apply. However, for infinitesimal changes $\alpha^{\star}$ will remain the same. Hence, the first term still vanishes completing our proof.

This result has interesting economic insights. First, the fact that the value of the investment opportunity decreases with $\beta$ is intuitive, since an increase in $\beta$ could be thought of as a decrease in the overall productivity of the investment opportunity, as for any given investment $\alpha \in\left[\alpha_{0}, 1\right)$ we are learning less than we did before. The reduction of value of the option is a logical consequence.

The effect of an increase of $\beta$ in the value of investment is less obvious. If the current solution is at an interior point and increases in $\beta$ makes marginal investment more productive than before $\left(\breve{\alpha}(\beta)<\alpha^{\star}(\beta)\right)$, then the firm has an incentive to devote more resources to the pilot project in order to take advantage of the shift in learning from the beginning to the end of the cumulative investment path. This is not inconsistent with the fact that the option value has decreased, since the value that the firm would obtain if the investment level is not increased in response to an increase in $\beta$ would be even less than the value of the option at the new optimal investment level. In other words we have that:

$$
V\left(\alpha^{\star}(\beta), \beta\right)>V\left(\alpha^{\star}\left(\beta^{\prime}\right), \beta^{\prime}\right)>V\left(\alpha^{\star}(\beta), \beta^{\prime}\right)
$$


This effect of an increase in investment as a response to a higher $\beta$ will be observed in most parameter combinations that yield interior point solutions. Nevertheless, the opposite effect is also possible, an increase in $\beta$ may decrease incentive to invest when $\breve{\alpha}(\beta)<\alpha^{\star}$. This occurs only when uncertainty is unusually high, $\beta$ is low and the lower boundary $\alpha_{0}$ is also unusually low. For those cases, the optimal investment level is low and increases in $\beta$ decrease marginal revenues. Less investment will result as a consequence. Although theoretically possible, this scenario shall not appear frequently in practical scenarios.

Finally, in case the current optimal investment is at the boundaries, small changes in $\beta$ would not have any effect in the investment decision unless $V\left(\alpha_{0}\right)$ is very close to $V(1)$. In this particular case, optimal investment may jump from $\alpha_{0}$ to 1 or viceversa as a result of changes in $\beta$. The direction of this jump depends on whether $\alpha_{0}$ exceeds $\breve{\alpha}(\beta)$ or not.

\subsection{Sensitivity to the Uncertainty Parameters $\left(\kappa, \sigma_{m}\right.$ and $\left.\sigma_{z}\right)$}

First let us analyze the consequences of changing the relative weight of technical uncertainty $\kappa$ keeping market uncertainty fixed. From equation (17) and a bit of algebra, it is easy to check that $\delta M R(\alpha) / \delta \kappa>0$ for any $\alpha \in[0,1]$ unless $\sigma_{m}$ takes unreasonably large values. Moreover, since the vega of an option is positive and the effective volatility is increasing in $\kappa$, by simple envelope arguments we can easily verify that the value of the option $V^{\star}$ is also increasing in $\kappa$ (see the appendix for algebraic details). This implies that the boundary values of $M R\left(\alpha_{0}\right)$ and $M R(1)$ are also increasing in $\kappa$ and, hence, the optimal fraction of investment will be nondecreasing in $\kappa, \delta \alpha^{\star} / \delta \kappa \geq 0$. This result is fairly intuitive since, as investment in the pilot stage is made to reduce technical uncertainty, the higher the $\kappa$ the bigger the relative weight of technical uncertainty in the project and the higher the total gain of reducing this technical uncertainty. This increases the optimal investment fraction if the solution was at an interior point. Increasing $\kappa$ has also an absolute effect, since it is equivalent to increasing the effective volatility term $\widehat{\sigma}(\alpha)$. As is well known, an increase in total uncertainty increases the value of an option due to the convexity of its payoff function.

Since $\kappa$ is directly proportional to $\sigma_{z}$, the previous argument also applies if we increase $\sigma_{z}$ keeping $\sigma_{m}$ constant. This increases total investment due to two effects: the first an absolute effect since increasing $\sigma_{z}$ amounts to an increase in total uncertainty and the second a relative effect since we are increasing the type of uncertainty that is reduced by investment. The value of the option $V^{\star}$ is also nondecreasing in $\sigma_{z}$.

An increase in $\sigma_{m}$ keeping $\sigma_{z}$ constant also increases the value of the option. Unlike for technical uncertainty optimal investment is non increasing in $\sigma_{m}$, unless the total effective volatility of the project is low (refer to the appendix for the exact threshold). This is also expected, since $\sigma_{m}$ is the uncertainty that is resolved by the passage of time, without the need for investment. This results in a disincentive to reduce the type of 
uncertainty that could be resolved by investment, technical uncertainty. Less investment in the development stage follows as a logical conclusion. These results are consistent with those obtained by Pindyck (1993) and Schwartz (2003). For the cases where $\widehat{\sigma}(\alpha)$ is low, raising $\sigma_{m}$ will increase the marginal gain from investing, thus, market and technical uncertainty have reinforcing effects on the incentive to invest. However, when effective volatility is low, the optimal decision is generally not to invest at all. Thus, changes in market uncertainty will have no effect in the optimal investment decision.

\subsection{Sensitivity to Assessments of Revenues and Costs $\left(S_{0}\right.$ and $\left.K_{0}\right)$}

We will start studying the effect on the value of the option and our optimal investment level $\alpha^{\star}$ of changes in $K_{0}$, the initial assessment of costs. An increase in $K_{0}$ will decrease the value of an option and a decrease in $K_{0}$ will increase it, as expected. However, the effect on the optimal investment decision depends on how the moneyness of the option affects its vega, the marginal gain due to an increase in volatility.

We have defined the moneyness of the option as the ratio $S_{0} e^{\left(\delta_{k}-\delta_{s}\right) T} / K_{0}$, which we will label $M$. An at the money option corresponds to $M=1$ and it has roughly the same chances of being exercised or not in the future. A high value of $M$ corresponds to an in the money option that is very likely to be exercised in the future, whereas a low $M$ corresponds to an out of the money option with a small probability of exercise.

The vega of an option is increasing in its moneyness when $d_{1}(\alpha)<0$, which is equivalent to $\log (M)<$ $-\widehat{\sigma}(\alpha)^{2} T / 2$. This corresponds to an out of the money option with low chances of being exercised, more so if the volatility is high. The vega of an option is decreasing in its moneyness when $d_{1}>0$, or $\log (M)>$ $-\widehat{\sigma}(\alpha)^{2} T / 2$. This corresponds to an in the money or a bit out of the money options with reasonable chances of being exercised. Translated to our real options scenario, if it is likely that commercial stage will be undertaken, the marginal gain from higher volatility decreases as this likelihood increases. Learning becomes less valuable as the chances of launching the commercial stage increase. When the option is out of the money, the contrary occurs. An increase in moneyness increases vega and makes learning more valuable. We could understand better this result if we think of why uncertainty increases the value of an option. It does so because it allows to profit from the successful outcomes while limiting losses on the negative side. When an option is deep in the money its value is close to the underlying minus the discounted strike price since it is very unlikely that it will not be exercised in the future. Additional increases in volatility although valuable, are less so than when the option is at the money and the final exercise decision is more uncertain. Therefore we have that if $d_{1}(\alpha)>0$ then $\delta M R(\alpha) / \delta K_{0}>0$ and viceversa. If these property holds for the current optimal solution $\alpha^{\star}$ then $\delta \alpha^{\star} / \delta K_{0} \geq 0$. An increase in the expected costs of a project when the project is likely to be launched decreases its moneyness and thus, increases the optimal level of investment, 
since it pays more to learn in the pilot stage. The reverse logic could be applied to an increase in $K_{0}$ for options that are way out of the money. Note that, even though an increase in the assessment of costs for a project likely to be undertaken is always bad news for the firm, since it decreases the value of its investment opportunity, it might be optimal to invest more in the development stage of this project in response to these bad news. Doing otherwise will decrease the value of the investment opportunity even further. This is similar to what we found when analyzing the sensitivity to $\beta$.

The effect on marginal revenues of an increase in $S_{0}$ has a similar economic explanation. From the appendix we can see that marginal revenues are non decreasing in $S_{0}$ if $d_{1}(\alpha)<\widehat{\sigma}(\alpha) \sqrt{T}$ or equivalently when $\log (M)<\widehat{\sigma}(\alpha)^{2} T / 2$. When there is a significant probability of not launching the commercial stage, increases in the assessment of its revenues make learning more valuable and result in a higher incentive to invest. On the contrary if $\log (M)>\widehat{\sigma}(\alpha)^{2} T / 2$ then marginal revenues are non increasing in $S_{0}$. In other words, if it is very likely that the commercial stage will be launched, an increase in expected revenues reduces the incentive to invest. Again, if these property holds for the current optimal solution $\alpha^{\star}$ we have that $\delta \alpha^{\star} / \delta S_{0} \leq 0$. Paralleling the reasoning made for costs, the optimal response to the good news of an increase in the expected revenues of a very likely to be exercised project is to reduce the investment in its development stage.

\subsection{Sensitivity to the Drift Terms of Revenues and Costs $\left(\delta_{s}\right.$ and $\left.\delta_{k}\right)$}

Effects of changes in the drift terms on the value of the option and the optimal investment policy are very similar to the ones occurring for changes in $S_{0}$ and $K_{0}$, as increases in $\delta_{k}$ are equivalent to decreases in $K_{0}$ and increases in $\delta_{s}$ to decreases in $S_{0}$. The same moneyness effect in the vega of the option that we analyzed extensively above will drive all the results. The value of the investment opportunity will always be non decreasing in $\delta_{k}$ and non increasing in $\delta_{s}$. However, optimal investment will be non increasing in $\delta_{k}$ if $d_{1}\left(\alpha^{\star}\right)>0$ and will be nondecreasing otherwise, i.e., if the option is well out of the money. For the case of $\delta_{s}$, optimal investment will only be nondecreasing in $\delta_{s}$ is the option is well in the money, more specifically if $d_{1}\left(\alpha^{\star}\right)>\delta_{s} \widehat{\sigma}\left(\alpha^{\star}\right) \sqrt{T}$, and will be non increasing otherwise.

\section{Robustness}

The model we developed gave us closed form solutions at an expense of some simplifying assumptions. These assumptions were made for simplicity and, as we will see in this section, most of them could be relaxed without significantly affecting our main results. Probably the only assumption whose relaxation could not be accommodated in this setting is the one period investment. Allowing for multiple revisions on the investment 
process across the pilot stage requires a discrete or continuous time dynamic programming formulation which is beyond the scope of this paper. In this section we will sketch the changes in the procedure and solutions to our problem as a result of incorporating the possibility of waiting after completion of the development stage, introducing a growth rate dependent on the level of investment and making time to completion flexible and proportional to the amount of resources devoted to the pilot stage.

\subsection{Incorporating the Possibility of Waiting after Completion}

Introducing this extra level of flexibility at the end of the pilot stage will change the boundary condition of the equation satisfied by $V(\alpha)$. Before, the commercial stage had either to be undertaken or abandoned, implying a value of the option at time $\mathrm{T}$ of $\max \left[S_{T}-K_{T}, 0\right]$. Instead of that, we know have get at time $\mathrm{T}$ a perpetual American option to exchange one asset for another with the process of $S_{t}$ and $K_{t}$ for $t \geq T$ following in the risk neutral measure:

$$
\begin{gathered}
d S_{t}=\left(r_{f}-\delta_{s}\right) S_{t} d t+\sigma_{s} S_{t} d w_{1} \\
d K_{t}=\left(r_{f}-\delta_{k}\right) K_{t} d t+\sigma_{k} K_{t} d w_{2}
\end{gathered}
$$

with $d w_{1}$ and $d w_{2}$ being two standard Brownian Motions with a correlation of $\rho$ in the risk neutral measure. Note that, since no more technical investment is made only the market Brownian $w_{2}$ is driving the value of costs. This setup could be easily solved by a further change of measure, considering $K_{t} e^{\delta_{k} t}$ as our new numeraire asset. We obtain that the commercial project will be launched if $S_{T}>K_{T} \frac{\gamma}{\gamma-1}$ where $\gamma$ is equal to:

$$
\gamma=1 / 2-\left(\delta_{k}-\delta_{s}\right) / \sigma_{M}^{2}+\sqrt{2 \delta_{k} / \sigma_{M}^{2}+\left[\left(\delta_{k}-\delta_{s}\right) / \sigma_{M}^{2}-1 / 2\right]^{2}}
$$

The value of the option at time $\mathrm{T}$ is equal to $S_{T}-K_{T}$ if $S_{T}>K_{T} \frac{\gamma}{\gamma-1}$. Otherwise it is given by: $(\gamma-$ 1) ${ }^{\gamma-1} S_{T}^{\gamma} K_{T}^{1-\gamma} / \gamma^{\gamma}$

This setting makes sense only when $\delta_{s}>\delta_{k}$ since otherwise the firm will want to wait forever.

By plugging this new boundary condition we could find the equivalent of (8) under this more flexible scenario. It is given by:

$$
V(\alpha)=S_{0} e^{-\delta_{S} T} N\left(d_{a}\right)-K e^{-\delta_{K} T} N\left(d_{b}\right)+(\gamma-1)^{\gamma-1} S_{0}^{\gamma} K_{0}^{1-\gamma} e^{\left(\delta_{k}(\gamma-1)-\delta_{S} \gamma+0.5 \widehat{\sigma}^{2}(\alpha) \gamma(\gamma-1)\right) T} N\left(d_{c}\right)-\alpha \bar{I}(21)
$$


where $d_{a}(\alpha), d_{b}(\alpha)$ and $d_{c}(\alpha)$ are given by:

$$
\begin{array}{r}
d_{a}(\alpha)=\frac{\ln \left(S_{0}(\gamma-1) / K_{0} \gamma\right)+\left(\delta_{k}-\delta_{s}+\widehat{\sigma}^{2}\right) T}{\widehat{\sigma} \sqrt{T}} \\
d_{b}(\alpha)=d_{a}(\alpha)-\widehat{\sigma}(\alpha) \sqrt{T} \\
d_{c}(\alpha)=\frac{\ln \left(K_{0} \gamma / S_{0}(\gamma-1)\right)-\left[\left(\delta_{k}-\delta_{s}\right) \gamma+\widehat{\sigma}^{2}(\alpha) \gamma(\gamma-1) / 2\right] T}{\gamma \widehat{\sigma}(\alpha) \sqrt{T}}
\end{array}
$$

with $\widehat{\sigma}(\alpha)$ being defined as before. The complete calculation is given in the appendix.

Obviously this expression is higher than the one obtained previously, with the difference being greater in the case of low $\delta_{s}$, i.e., little opportunity cost of waiting and high market uncertainty, i.e, high benefits from waiting. The sensitivity of changes in $\beta$, the uncertainty parameters and moneyness parameters in the optimal investment policy will have the same qualitative effects as in our previous case, but these effects will be less strong, since now technical uncertainty plays a relatively less important role against market uncertainty than in the benchmark model.

\subsection{Incorporating an Investment Dependent Growth Rate}

In our main model, pilot investment is only affecting the technical volatility of the costs process. Nevertheless, it is common that these technological investments also bring together a expected cost reduction.

Using the same proportionality assumption as for the technical volatility term, we can assume the drift term $\alpha_{k}(I)$ has the following form:

$$
\alpha_{k}(I)=\alpha_{k}-\nu\left(\frac{I}{\bar{I}}\right)^{2 \eta}
$$

with $\alpha_{k}$ and $\nu>0$ being constant real numbers. We can think of $\alpha_{k}$ as the a component of the drift determined by market factors and independent on the level of investment. The second term is the expected rate of cost reduction inherent to the technology under consideration and is only present when investment is taking place. There is a proportionality relation between the level of pilot investment and the contribution to expected cost reduction from this second term, with the proportionality coefficient given by $\eta$. As an increase in investment will generally reduce expected costs, $\nu$ is assumed to be a positive constant.

The value of the option to invest is still given by (8), but with $\delta_{k}(\alpha)=\delta_{k}+\nu \alpha^{2 \beta}$ replacing $\delta_{k}$ in (8), (9) and (10).

As the key driver of all our results was the marginal revenue function, we need to recalculate with these additional drift effect. Let us label the new marginal revenue function by $\widehat{M R}(\alpha)$ to distinguish it from the 
marginal revenue function of the previous model $M R(\alpha)$. It is given by:

$$
\widehat{M R}(\alpha)=M R(\alpha)+\frac{\delta V(\alpha)}{\delta \delta_{k}} \frac{\delta \delta_{k}(\alpha)}{\delta \alpha}
$$

The sensitivity of the option to invest to changes in $\delta_{k}$ is given by:

$$
\frac{\delta V(\alpha)}{\delta \delta_{k}}=T K_{0} e^{-\left(\delta_{k}+\nu \alpha^{\beta}\right) T} N\left(d_{2}(\alpha)\right)
$$

Thus, the adjusted Marginal Revenue function is given by

$$
\widehat{M R}(\alpha)=S_{0} e^{-\delta_{S} T} N^{\prime}\left(d_{1}(\alpha)\right) \sqrt{T} \beta \frac{\kappa \sigma_{m}}{\sqrt{1+\kappa \alpha^{2 \beta}}} \alpha^{2 \beta-1}+T K_{0} e^{-\left(\delta_{k}+\nu \alpha^{\beta}\right) T} N\left(d_{2}(\alpha)\right) \nu \eta \alpha^{\eta-1}
$$

This additional drift effect in the marginal gains from investing is positive, due to the fact that the value of the option to invest is non decreasing in $\delta_{k}$ and to our assumption that $\nu>0$. Investing is not only valuable from the learning that results but also for the fact that it decreases costs in expectation. This results in an extra incentive to invest compared to the benchmark model analyzed in Section 4 and the insights and conclusions given there need to be adjusted to incorporate this extra effect. $\eta$ will govern the way in which expected cost reduction due to technical investment is distributed along the cumulative investment path and $\nu$ is a measure of the strength of the expected cost reduction.

\subsection{Incorporating Variable Time to Completion}

In our main model we made the assumption that time to completion was fixed regardless of the level of investment. However, more resources devoted to the pilot stage may increase time to completion in many cases. We will incorporate this additional effect by making $T$ an increasing function of our fraction invested $\alpha$. Assume this relation takes the general form $T(\alpha)$ with, $T(1)=T, T\left(\alpha_{0}\right)=T_{0}$ and $T(\alpha)$ increasing in $\alpha$. $T_{0}$ is of course the minimum time in which the pilot can be completed. For instance, in case time is linearly proportional to investment, $T(\alpha)$ could take the particular form:

$$
T(\alpha)=T-\frac{T-T_{0}}{1-\alpha_{0}}(1-\alpha)
$$

Now, the value of our option given a fixed investment $\alpha, V(\alpha)$ is still given by (8) with $T(\alpha)$ replacing our previously fixed $\mathrm{T}$. Let's call the new marginal revenue $\widetilde{M R}(\alpha)$. It is given by:

$$
\widetilde{M R}(\alpha)=M R(\alpha)+\frac{\delta V}{\delta T(\alpha)} \frac{\delta T(\alpha)}{\delta \alpha}
$$


Since $\delta T(\alpha) / \delta \alpha>0$ the additional effect on the marginal revenue function will decrease the previous marginal revenues function if $\delta V / \delta T(\alpha)<0$, in other words if the theta ${ }^{2}$ of the option is negative, and will increase $M R(\alpha)$ otherwise.

The theta of the option to invest is given by:

$$
\frac{\delta V(\alpha, T)}{\delta T}=\frac{S_{0} N^{\prime}\left(d_{1}(\alpha)\right) \widehat{\sigma}(\alpha) e^{-\delta_{s} T}}{2 \sqrt{T}}-\delta_{s} S_{0} e^{-\delta_{s} T} N\left(d_{1}(\alpha)\right)+\delta_{k} K_{0} e^{-\delta_{k} T} N\left(d_{2}(\alpha)\right)
$$

The value of the investment opportunity will be decreasing in time to completion in case $\delta_{s}$ (the opportunity cost of revenue forgone) or $S_{0} / K_{0}$ are significant. In this case, the firm should launch the commercial stage as soon as it is feasible to do so, as delaying time to completion results in a significant economic loss. When $\delta_{k}$ is high, increasing time to completion may actually increase the value of the option, as it allows for a greater expected reduction on final costs.

In most competitive market environments $\delta_{s}$ is considerable, so that speeding up completion of the development stage brings economic value to the firm. In this case, the marginal gain from learning is reduced from that obtained in model of Section 2, as additional investment has the extra negative effect of delaying the launch of the pilot project. Thus, all the results and insights of the previous section should be adjusted to account for this extra disincentive to invest.

\section{Applications to Supply Chain Management}

Opportunities to invest in order to learn certain features about a product or project without committing all the resources required for its full scale implementation are commonly present in different industries and across different stages of the supply chain of a firm. Market uncertainty will generally impact the value of any project all the way from its initial conception to the end of its lifetime. However, idiosyncratic uncertainty, could be thought of as a fixed and time independent source of risk that could be reduced by certain actions taken by the firm and, whose risk, once eliminated, will not affect the value of the project thereafter.

Both sources of risk need not only be present at the development stage of a project, but also in the procurement, marketing and production stages. We believe that the general theoretical framework developed in the previous sections could be applied, with some necessary modifications to account for the specificity of the investment under consideration, to help decision making in many practical business situations. Below we provide a few examples.

\footnotetext{
${ }^{2}$ In the financial jargon theta denotes the sensitivity of the price of an option to the passage of time
} 
1. Airplane Manufacturing Industry: Developing a new model of commercial aircraft

Given the complexity involved in the final product, developing a new airplane model is a gigantic task that demands an enormous amount of technical, financial and administrative resources. Moreover, the value of the revenue stream to be obtained during the lifetime of a future airplane model depends ultimately on the worldwide demand for plane tickets. Hence, it is heavily affected by the current and expected future state of the economy. In addition, as a new jumbo model usually comes together with marginal or dramatic improvements of existing technological systems, there is significant uncertainty with respect to the efficiency of these new technologies, and thus, with respect to the final costs of the project, which are also influenced by market factors. Simulation and related engineering processes allow testing of many of these technologies without the need to implement them completely. Technical pilot projects play a pivotal role in the development stage by helping reduce the technical uncertainty associated with the final implementation and recurrent costs of the new model under consideration.

2. Mining and Oil Industry: Exploring new reserves

The opportunity to exploit a new mine or oil well presents considerable uncertainty with regard to the final level of reserves that may be available for extraction. Market factors will drive the price of the commodity to be obtained and the geological uncertainty will govern the extraction rate of the firm once the mine or oil well becomes operative. Both, market and geological uncertainty will affect the value of the mine or well, but the latter is fixed and independent of time and market considerations. Hence, it plays the role of the idiosyncratic uncertainty in our model. Firms in these industries invest heavily in geological testing that reduce risk with respect to the level of reserves without engaging in expensive exploration activities. The present framework should help determine the optimal investment level for these activities.

3. Fast Food Industry: Introducing mature products in new markets.

This is a natural consequence of globalization, multinationals firms are able to expand into new markets with strikingly different characteristics from the markets they are used to serving. The opening of many American fast food chains in the Chinese market in the last 10 years is one of the first examples that comes to mind. Cultural, social, religious or biological factors could greatly influence the value that a customer derives from a product or service. Launching the product in a smaller market with similar characteristics to the market to be targeted is an alternative frequently considered. For example, Thailand, Taiwan or the Philippines may provide appropriate proxies for the Chinese market. We could think of the purchasing power of consumers in the new market as the tradable market uncertainty, since 
it is changing to time and it is correlated with economic fundamentals, such as growth or unemployment rates. However, the value that a consumer derives from a product and his intrinsic willingness to pay are inherent to the consumers and, in general, to the market in question. This value is also uncertain and it is reasonable to consider it fixed or changing very slowly with time. We can consider uncertainty about the value that a product will have for consumers in the new market as the idiosyncratic uncertainty in our model. Therefore, the problem of how much to invest in the trial launch of a new product may be handled by appropriately modifying our setting. For this particular case we need the revenues, instead of the costs, to be driven by market and private uncertainty.

4. Auto Industry: Contracting with a new supplier.

Efficient procurement is one of the most critical processes in the auto industry, as hundreds of parts are needed to assemble the final product. Although long standing relationships between the auto manufacturing companies and their suppliers are common in the industry, the option of obtaining key components from new suppliers also appears frequently. Moreover, it is not uncommon that these procurement possibilities come together with significantly lower acquisition costs. However, service quality, is equally or more important to the auto companies than the final costs. Hence, their satisfaction depends significantly in non quantifiable characteristics that are usually difficult to include in a contract such as responsiveness, flexibility or coordination, to cite a few. There is considerable risk with respect to how an unknown supplier will behave along these dimensions. Changing the entire procurement processes to work mainly with a new firm instead of another may require a significant irreversible investment, as many procedures, facilities and other resources may need to be adjusted or altered to work with the potential new supplier. It is frequently the case, that auto companies start testing potential new suppliers by awarding them quantities that are not critical for its overall performance before engaging with them on a long term basis. This uncertainty with respect to the non quantifiable service levels could be thought of as a fix source of risk that it is intrinsic to the new firm, and determined by factors such as its corporate culture, its know-how or the personal skills of its people, among others. Hence, it is equivalent to the idiosyncratic technical uncertainty in our model and could be partially resolved with these trial procurement contracts. Thus, our setting could be stretched to analyze what is the optimal level of resources that should be devoted to set up these contracts. 


\section{Conclusions}

We have developed a theoretical framework to value pilot project investments in which the revenues for the commercial stage are driven by tradable market uncertainty and costs are driven by both, market and idiosyncratic technical uncertainty. The evolution of technical uncertainty is modeled by a learning coefficient that establishes a proportionality relationship between cumulative investment and total uncertainty resolved in the pilot stage. For the case of decreasing returns to investment an interior point solution to the investment problem may be obtained: the total budget assigned to the development stage is somewhere between the minimum needed for its completion and the maximum budgetary bound. In this case valuable economic implications are derived from our model. First, an increase in the learning coefficient, which is equivalent to reducing total learning for any given investment level, generally raises optimal investment in the pilot stage as it increases the contribution to learning from later dollars invested. Secondly, although increases in both, market and technical uncertainty, increase the value of the investment opportunity, they have different effects in the optimal investment decision. An increase in the latter result in an incentive to invest, while increases in the former generally reduce the optimal investment in the development stage. In addition, and perhaps a bit surprisingly, when it is not very likely that the commercial stage will be discarded, an increase in the assessment of costs, although bad news for the manager, increases the amount of resources that should be devoted to learning. Analogous effects were found for changes in the assessment of revenues and the drift rates of revenues and costs.

Some relaxations to the main assumptions of the model were incorporated and its effects on the results and conclusions were analyzed: the possibility of waiting after completion of the pilot stage, a general drift rate dependent on the investment made and variable time to completion. They all introduce additional effects to the marginal revenue function that we need to incorporate to our previous analysis. As we have limited the scope of this paper to a one period investment setting, a general continuous time control problem is left for future work. Other possibilities for future research include analyzing how investment should be distributed between a portfolio of competing and substitute technologies, or focusing on the learning in the commercial stage itself, which in the current framework is treated as a black box.

Stanford, California

February, 2005 


\section{Appendix}

\subsection{Change of Measure}

We will work with a standard 3 dimensional Brownian motion $B_{t}$ defined in the standard probability space $(\Omega, \mathfrak{F}, P)$. The information set at time $\mathrm{t}$ will be given by the filtration defined by this Brownian motion. Furthermore, $B_{t}=\left(w_{1}, w_{2^{\prime}}, z\right)$, with $w_{2^{\prime}}$, defined as $\left(w_{2}-\rho w_{1}\right) /\left(\sqrt{1-\rho^{2}}\right)$, being the market Brownian for costs that is orthogonal to the market Brownian of revenues.

From Assumptions 4 and 5, the market prices of risk of $w_{1}$ and $w_{2}$ are given by $\lambda_{1}=\left(\alpha_{s}+\delta_{s}-r_{f}\right) / \sigma_{s}$ and $\lambda_{2}=\left(\alpha_{c}+\delta_{c}-r_{f}\right) / \sigma_{c}$ respectively. Furthermore, since Assumption 7 implies a zero market price of technical risk then $\lambda_{z}=0$. This implies, as we will see later, that the technical volatility term will have no effect in the drift when switching from the real to the risk neutral probability measure.

By following standard arguments in the finance literature we can now construct and define the equivalent risk neutral measure $Q$ that will give us a value for our option to invest consistent with Assumptions 1-7. We will be working in this measure for the remaining of the paper.

Since $w_{2}=\rho w_{1}+\sqrt{1-\rho^{2}} w_{2^{\prime}}$ we can rewrite the process for $c_{t}$ as:

$$
d c_{t}=\alpha_{c} c_{t} d t+\rho \sigma_{c} c_{t} d w_{1}+\sqrt{1-\rho^{2}} \sigma_{c} c_{t} d w_{2^{\prime}}
$$

we can now redefine the new risk premium for $w_{2^{\prime}}$, which we will denote as $\lambda_{2^{\prime}}$ as the solution to:

$$
\alpha_{c}+\delta_{c}-r_{f}=\lambda_{1} \rho \sigma_{c}+\lambda_{2^{\prime}} \sqrt{1-\rho^{2}} \sigma_{c}
$$

which simplifies to:

$$
\lambda_{2^{\prime}}=\frac{\alpha_{c}+\delta_{c}-r_{f}-\rho \sigma_{c}\left(\alpha_{s}+\delta_{s}-r\right) / \sigma_{s}}{\sigma_{c} \sqrt{1-\rho^{2}}}
$$

Following standard arguments in the finance literature we construct our equivalent measure as follows:

Let $\theta=\left(\lambda_{1}, \lambda_{2^{\prime}}, \lambda_{z}\right)$ be the vector of market prices of risk corresponding to the three orthogonal Brownian motions. Let our density process $\xi_{t}$ be defined as:

$$
\xi_{t}=\exp \left(-\int_{0}^{t} \theta d B_{s}-\frac{1}{2} \int_{0}^{t} \theta . \theta d s\right)
$$


By Ito's Lemma it is easy to verify that $d \xi_{t}=-\xi_{t} \theta d B_{t}$. Moreover, since our market price of risk $\theta$ is bounded, $\xi_{t}$ is a martingale ${ }^{3}$ with finite variance and, therefore, the density process of an equivalent probability measure $Q$, which we defined by $\frac{d Q}{d P}=\xi_{t}$.

Then by Girsanov's Theorem, a standard Brownian Motion under $Q, B_{t}^{Q}$, is defined by $B_{t}^{Q}=$ $\left(w_{1 t}^{Q}, w_{2^{\prime} t}^{Q}, z_{t}^{Q}\right)=B_{t}+\theta d t=\left(w_{1 t}+\lambda_{1} t, w_{2^{\prime} t}+\lambda_{2^{\prime}} t, z_{t}+\lambda_{z} t\right)$. Replacing this expressions in (1) and (3) we get that the processes for $S_{t}$ and $K_{t}$ under $Q$ are given by:

$$
\begin{aligned}
d S_{t} & =\alpha_{s} S_{t} d t+\sigma_{s} S_{t}\left[d w_{1}^{Q}-\lambda_{1} d t\right]=\left(r_{f}-\delta_{s}\right) S_{t} d t+\sigma_{s} S_{t} d w_{1}^{Q} \\
d K_{t} & =\alpha_{k} K_{t} d t+\rho \sigma_{k} K_{t}\left[d w_{1}^{Q}-\lambda_{1} d t\right]+\sqrt{1-\rho^{2}} \sigma_{k} K_{t}\left[d w_{2^{\prime}}^{Q}+\lambda_{2^{\prime}} d t\right]+\sigma_{z}(I / \bar{I})^{\beta}\left[d z^{Q}+\lambda_{z} d t\right]
\end{aligned}
$$

Define $w_{2}^{Q}$ as a Brownian motion that has a correlation of $\rho$ with $w_{1}^{Q}$ and is independent from $z$. Replacing in the previous expressions the values for $\lambda_{1}, \lambda_{2^{\prime}}$ and $\lambda_{z}=0$, we can simplify the process followed by $S_{t}$ and $K_{t}$ to:

$$
\begin{aligned}
d S_{t} & =\left(r_{f}-\delta_{s}\right) S_{t} d t+\sigma_{s} S_{t} d w_{1}^{Q} \\
d K_{t} & =\left(r_{f}-\delta_{k}\right) K_{t} d t+\sigma_{k} K_{t} d w_{2}^{Q}+\sigma_{z}(I / \bar{I})^{2 \beta} K_{t} d z^{Q}
\end{aligned}
$$

with $\delta_{k}$ defined by:

$$
\delta_{k}=r_{f}-\alpha_{k}+\frac{\alpha_{c}+\delta_{c}-r}{\sigma_{c}} \sigma_{k}
$$

\subsection{Calculation of Equations (8) and (21)}

Under the risk neutral measure $Q, S_{t}$ and $K_{t}$ follow (5) and (6) respectively. The boundary condition corresponding to $(8)$ is given by:

$$
V(\alpha, T)=\max \left[S_{T}-K_{T}, 0\right]
$$

From section 5.1 we have that the boundary condition corresponding to (21) it is given by:

$$
V(\alpha, T)=\frac{(\gamma-1)^{\gamma-1} S_{T}^{\gamma} K_{T}^{1-\gamma}}{\gamma^{\gamma}} 1_{\left\{S_{T}<K_{T} \gamma /(1-\gamma)\right\}}+\left(S_{T}-K_{T}\right) 1_{\left\{S_{T}>K_{T} \gamma /(1-\gamma)\right\}}
$$

\footnotetext{
${ }^{3}$ Actually, Novikov's condition, i.e., $E\left[\exp \left(\frac{1}{2} \int_{0}^{T} \theta_{s} \cdot \theta_{s} d s\right)\right]<\infty$ is a much weaker requirement for $\xi_{t}$ to be a martingale and accommodates stochastic or time dependent market prices of risk. See Duffie (1996) for technical details. This condition is automatically satisfied in our setting since we will work with constant market prices of risk.
} 
where $\gamma$ is given by $(20)$.

We proceed to derive expression (21). The calculation for (8) is analogous and simpler. Let us denote the value at time $\mathrm{t}$ of the option to invest once the fixed payment has already been made by $\hat{V}(\alpha, t)$. Equivalently $V(\alpha, t)=\hat{V}(\alpha, t)-\alpha \bar{I}$ respectively. Once the initial investment has been made we could value this resulting option using the equivalent martingale measure principles from financial theory. The value of the investment opportunity at time 0 is given by:

$$
\hat{V}(\alpha, 0)=E^{Q}\left[e^{-r_{f} T}\left(S_{T}-K_{T}\right) ; S_{T}>K_{T} \frac{\gamma}{1-\gamma}\right]+E^{Q}\left[e^{-r_{f} T} \frac{(\gamma-1)^{\gamma-1} S_{T}^{\gamma} K_{T}^{1-\gamma}}{\gamma^{\gamma}} ; S_{T}<K_{T} \frac{\gamma}{1-\gamma}\right]
$$

where $\mathrm{Q}$ is the risk neutral measure. In order to simplify the calculation we will do a further change of numeraire and work in a risk neutral world with respect to $K_{t} e^{\delta_{k} t}$. The value of the option in units of $K_{t} e^{\delta_{k} t}$ will turn out to be a martingale in our $K_{t}$ risk neutral measure which we will label $R$. We will denote our discounted asset $S_{t} / K_{t}$ by $\widetilde{S}_{t}$. By a simple application of Ito's Lemma, under $R, \widetilde{S}_{t}$ follows the following diffusion:

$$
d \widetilde{S}_{t}=\left(\delta_{k}-\delta_{s}\right) \widetilde{S}_{t} d t+\widehat{\sigma}(\alpha) \widetilde{S}_{t} d w^{R}
$$

with $\widetilde{S}_{0}=S_{0} / K_{0}$ and $d w^{R}$ being a Standard Brownian Motion under our measure $R$. Now we can value the investment opportunity by pricing our option in a risk neutral world with respect to $K_{t} e^{\delta_{k} t}$. Since $\hat{V}(\alpha, t) e^{-\delta_{k} t} / K_{t}$ is a martingale under $R$ we have:

$$
\begin{gathered}
\frac{\hat{V}(\alpha, 0)}{K_{0}}=E^{R}\left[\frac{V(\alpha, T)}{K_{T} e^{\delta_{k} T}}\right] \\
\frac{\hat{V}(\alpha, 0)}{K_{0}}=E^{R}\left[e^{-\delta_{k} T}\left(\widetilde{S}_{T}-1\right) ; \widetilde{S}_{T}>\frac{\gamma}{1-\gamma}\right]+E^{R}\left[e^{-\delta_{k} T} \frac{(\gamma-1)^{\gamma-1} \widetilde{S}_{T}^{\gamma}}{\gamma^{\gamma}} \widetilde{S}_{T}<\frac{\gamma}{1-\gamma}\right] \\
\frac{\hat{V}(\alpha, 0)}{K_{0}}=E^{R}\left[e^{-\delta_{k} T}\left(\widetilde{S}_{T}-1\right) ; \widetilde{S}_{T}>\frac{\gamma}{1-\gamma}\right]+E\left[e^{-\delta_{k} T} \frac{(\gamma-1)^{\gamma-1} \widetilde{S}_{T}^{\gamma}}{\gamma^{\gamma}} \widetilde{S}_{T}<\frac{\gamma}{1-\gamma}\right] \\
\frac{\hat{V}(\alpha, 0)}{K_{0}}=E^{R}\left[e^{-\delta_{k} T} \widetilde{S}_{T} ; \widetilde{S}_{T}>\frac{\gamma}{1-\gamma}\right]-e^{-\delta_{k} T} P^{R}\left[\widetilde{S}_{T}>\frac{\gamma}{1-\gamma}\right]+E^{R}\left[e^{-\delta_{k} T} \frac{(\gamma-1)^{\gamma-1} \widetilde{S}_{T}^{\gamma}}{\gamma^{\gamma}} \widetilde{S}_{T}<\frac{\gamma}{1-\gamma}\right] \\
\frac{\hat{V}(\alpha, 0)}{K_{0}}=\widetilde{S}_{0} e^{-\delta_{s} T} N\left(d_{a}(\alpha)\right)-e^{-\delta_{k} T} N\left(d_{b}(\alpha)\right)+\frac{(\gamma-1)^{\gamma-1}}{\gamma^{\gamma}} \widetilde{S}_{0}^{\gamma} e^{\left(\delta_{k}(\gamma-1)-\delta_{S} \gamma+0.5 \widetilde{\sigma}^{2}(\alpha) \gamma(\gamma-1)\right) T} N\left(d_{c}(\alpha)\right)
\end{gathered}
$$


with $d_{a}(\alpha), d_{b}(\alpha)$ and $d_{c}(\alpha)$ given by:

$$
\begin{gathered}
d_{a}(\alpha)=\frac{\ln \left(S_{0}(\gamma-1) / K_{0} \gamma\right)+\left(\delta_{k}-\delta_{s}+\widehat{\sigma}(\alpha)^{2}\right) T}{\widehat{\sigma}(\alpha) \sqrt{T}} \\
d_{b}(\alpha)=d_{a}(\alpha)-\widehat{\sigma}(\alpha) \sqrt{T} \\
d_{c}(\alpha)=\frac{\ln \left(K_{0} \gamma / S_{0}(\gamma-1)\right)-\left[\left(\delta_{k}-\delta_{s}\right) \gamma+\widehat{\sigma}^{2}(\alpha) \gamma(\gamma-1) / 2\right] T}{\gamma \widehat{\sigma}(\alpha) \sqrt{T}}
\end{gathered}
$$

Multiplying the last expression by $K_{0}$ and substracting the certain payment of $\alpha \bar{I}$ we get our final result:

$V(\alpha, 0)=S_{0} e^{-\delta_{s} T} N\left(d_{a}\right)-K_{0} e^{-\delta_{k} T} N\left(d_{b}\right)+(\gamma-1)^{\gamma-1} S_{0}^{\gamma} K_{0}^{1-\gamma} e^{\left(\delta_{k}(\gamma-1)-\delta_{S} \gamma+0.5 \widehat{\sigma}^{2}(\alpha) \gamma(\gamma-1)\right) T} N\left(d_{c}\right)-\alpha \bar{I}$

\subsection{Proof of Proposition 4.1}

Let us first calculate the function $\frac{\delta M R(\alpha)}{\delta \beta}<0$. It is equal to:

$\frac{\delta M R(\alpha)}{\delta \beta}=\frac{S_{0} e^{-\delta_{S} T} \kappa \sigma_{m} \sqrt{T} N^{\prime}\left(d_{1}(\alpha)\right) \alpha^{2 \beta-1}}{\left(1+\kappa \alpha^{2 \beta}\right)^{3 / 2}}\left[\left(1+\kappa \alpha^{2 \beta}\right)(1+2 \beta \ln \alpha)-\kappa \alpha^{2 \beta} \beta \ln \alpha+d_{1}(\alpha) d_{2}(\alpha) \kappa \alpha^{2 \beta} \beta \ln \alpha\right]$

Hence, the sign of the derivative depends on the sign of the term inside the brackets. Let's label this term $f(\alpha)$. We can easily see that $f(1)>0$ and that $\lim _{\alpha \rightarrow 0} f(\alpha)=-\infty$. This implies that there exists at least a one zero for the function in the interval $(0,1)$, an $\breve{\alpha}(\beta)$ such that $f(\breve{\alpha}(\beta))=0$. Equivalently $\breve{\alpha}(\beta)$ solves the following equation:

$$
\frac{\ln (1 / \alpha)\left[2 \beta+\kappa \beta \alpha^{2 \beta}\left(1+d_{1} d_{2}\right)\right]}{1+\kappa \alpha^{2 \beta}}=1
$$

Uniqueness follows by showing that $f^{\prime}(\alpha)>0$ whenever $f(\alpha)=0$.

\subsection{Main Comparative Statics}

\subsubsection{Sensitivity to $\kappa$ keeping $\sigma_{m}$ fixed}

$$
\frac{\delta M R(\alpha)}{\delta \kappa}=\frac{S_{0} e^{-\delta_{s} T} \sqrt{T} \beta \sigma_{m} \alpha^{2 \beta-1} N^{\prime}\left(d_{1}(\alpha)\right)\left[\kappa \alpha^{2 \beta}\left(1+d_{1}(\alpha) d_{2}(\alpha)\right)+2\right]}{2\left(1+\kappa \alpha^{2 \beta}\right)^{3 / 2}}
$$

This derivative is positive if and only if: $\kappa \alpha^{2 \beta}\left(1+d_{1}(\alpha) d_{2}(\alpha)\right)+2>0$. Since $d_{1}(\alpha) d_{2}(\alpha)$ is decreasing in volatility and hence in $\alpha$ then $\kappa\left(1+d_{1}(1) d_{2}(1)\right)+2>0$ suffices for this to hold for $\alpha \in[0,1]$. Unless $\sigma_{m}$ takes unusually high values $\delta M R(\alpha) / \delta \kappa>0$. By the envelope condition the value of the option is nondecreasing in $\kappa$ since $\delta V(\alpha, \kappa) / \delta \kappa>0$. 


\subsubsection{Sensitivity to $\sigma_{m}$ keeping $\sigma_{z}$ fixed}

$$
\frac{\delta M R(\alpha)}{\delta \sigma_{m}}=\frac{S_{0} e^{\delta_{S} T} \sqrt{T} \sigma_{Z}^{2} \beta \alpha^{2 \beta-1} N^{\prime}\left(d_{1}(\alpha)\right)\left[d_{1}(\alpha) d_{2}(\alpha)-1\right]}{\left(\sigma_{M}^{2}+\sigma_{Z}^{2} \alpha^{2 \beta}\right)^{3 / 2}}
$$

which is negative unless, $\widehat{\sigma}(\alpha)^{2} T+\widehat{\sigma}(\alpha)^{4} T^{2} / 4<\left(\ln \left(S_{0} / K_{0}\right)+\left(\delta_{k}-\delta_{s}\right) T\right)^{2}$. This condition requires low values for the total effective volatility. The value of the option, $V^{\star}$ is always nondecreasing in market uncertainty since $\delta V\left(\alpha, \sigma_{M}\right) / \delta \sigma_{M}>0$.

\subsubsection{Sensitivity to $K_{0}$}

$$
\frac{\delta M R(\alpha)}{\delta K_{0}}=\frac{S_{0} e^{\delta_{S} T} \kappa \beta \alpha^{2 \beta-1} d_{1}(\alpha) N^{\prime}\left(d_{1}(\alpha)\right)}{K_{0}\left(1+\kappa \alpha^{2 \beta}\right)}
$$

Marginal revenues are nondecreasing in $K_{0}$ if $d_{1}(\alpha)>0$ and are non increasing in $K_{0}$ otherwise. This condition holds if and only if $\ln \left(S_{0} / K-0\right)+\left(\delta_{k}-\delta_{s}\right) T>\widehat{\sigma}(\alpha)^{2} T / 2$, in other words, for an out of the money option, more out of the money when the volatility is high.

The value of the option $V^{\star}$ is always decreasing in $K_{0}$. This holds by the envelope condition and the fact that $\delta V\left(\alpha, K_{0}\right) / \delta K_{0}<0$.

\subsubsection{Sensitivity to $S_{0}$}

$$
\frac{\delta M R(\alpha)}{\delta S_{0}}=\frac{e^{-\delta_{S} T} \kappa \beta \alpha^{2 \beta-1} N^{\prime}\left(d_{1}(\alpha)\right)\left(\sigma_{m} \sqrt{1+\kappa \alpha^{2 \beta}} \sqrt{T}-d_{1}(\alpha)\right)}{\sqrt{1+\kappa \alpha^{2 \beta}}}
$$

Marginal revenues are nondecreasing in $S_{0}$ if $d_{1}(\alpha)<\sigma_{M} \sqrt{1+\kappa \alpha^{2 \beta}} \sqrt{T}$ and are non increasing in $S_{0}$ otherwise.

The value of the option $V^{\star}$ is always increasing in $S_{0}$. This holds by the envelope condition and the fact that $\delta V\left(\alpha, S_{0}\right) / \delta S_{0}>0$.

\subsubsection{Sensitivity to $\delta_{k}$}

$$
\frac{\delta M R(\alpha)}{\delta \delta_{k}}=-\frac{S_{0} e^{-\delta_{S} T} \kappa \beta \alpha^{2 \beta-1} d_{1}(\alpha) N^{\prime}\left(d_{1}(\alpha)\right) T}{1+\kappa \alpha^{2 \beta}}
$$

The effect is similar to the sensitivity to $K_{0}$. Marginal revenues are nondecreasing in $\delta_{k}$ if $d_{1}(\alpha)<0$ and are non increasing in $K_{0}$ otherwise. By the same previous arguments the value of the option $V^{\star}$ is always nondecreasing since $\delta V\left(\alpha, \delta_{k}\right) / \delta \delta_{k}>0$. 


\subsubsection{Sensitivity to $\delta_{s}$}

$$
\frac{\delta M R(\alpha)}{\delta \delta_{s}}=\frac{S_{0} \kappa \beta \alpha^{2 \beta-1} \sqrt{T} e^{-\delta_{s} T} N^{\prime}\left(d_{1}(\alpha)\right)\left[d_{1}(\alpha) \sqrt{T}-\delta_{s} \sigma_{m} \sqrt{1+\kappa \alpha^{2 \beta}}\right]}{1+\kappa \alpha^{2 \beta}}
$$

The effect is similar to the sensitivity to $S_{0}$. Marginal revenues are nondecreasing in $\delta_{s}$ if $d_{1}(\alpha)>$ $\delta_{s} \sigma_{m} \sqrt{1+\kappa \alpha^{2 \beta}} / \sqrt{T}$ and are non increasing in $\delta_{s}$ otherwise. By the same previous arguments the value of the option $V^{\star}$ is always non increasing in $\delta_{s}$ since $\delta V\left(\alpha, \delta_{s}\right) / \delta \delta_{s}<0$. 


\section{References}

[1] Brach M. and Paxson D., A Gene to Drug Venture: Poisson Options Analysis, RE्SD Management, vol 31 No 2 203-214, 2001.

[2] Cortazar G., et. al, Optimal Exploration Investments under Price and Geological-Technical Uncertainty: a real options model, RED Management, v31 No 2 181-189, 2001.

[3] Duffie D., Dynamic Asset Pricing Theory, Princeton University Press, 2001.

[4] Dixit, A and Pindyck, R., Investment Under Uncertainty, Princeton University Press, 1994.

[5] Errais E. and Sadowsky, J., Valuing Pilot Project Investments in Incomplete Markets: A Compound Option approach, Working Paper Stanford University, 2005.

[6] Grossman G. and Shapito C., Optimal Dynamic R\&D Programs, Rand Journal of Economics, Vol 17 581-593, 1986.

[7] Hull John C., Options, Futures and Other Derivatives, Prentice Hall, 2003.

[8] Lucas R. E. Jr., Optimal Management of a Research and Development Project, Management Science, Vol 17 No 11 679-697, 1971.

[9] Margrabe W., The Value of an Option to Exchange One Asset for Another, Journal of Finance, $33,177-86(1978)$

[10] Majid S. and Pyndick R. S., Time to Build, Option Value and Investment Decisions, Journal of Financial Economics 18, 7-27, 1987.

[11] Merton R.C.,Theory of rational option pricing. Bell Journal of Economics and Management Science 4, 141-183, 1973.

[12] Messica A. and David I., Optimal expenditure patterns for risky R\&D projects with time dependent returns, RESD Management, vol 30 No. 3 247-253, 2000.

[13] McDonald R. and Siegel D., The Value of Waiting to Invest, Quarterly Journal of Economics, 101 707-728, 1986.

[14] Pyndick R. S., Investments of Uncertain Cost, Journal of Financial Economics 34, 53-76, 1993.

[15] Roberts K. and Weitzman M. L. , Funding Criteria for Research, Development and Exploration Project, Econometrica 49 p. 1261-1288, 1981.

[16] Schwartz E. and Zoraya C., Investment under uncertainty in Information Technology, Acquisition and Development Projects, Management Science, vol 49 57-70, 2003. 